\title{
FATHOM
}

\section{Les romans du Wessex, une esthétique du regard}

Le Way-Sex, un éros visuel, de la fem-être à la tombe ou un trajet nommé désir

\section{Annie Escuret-Bertrand}

\section{(2) OpenEdition}

\section{Journals}

Édition électronique

URL : http://journals.openedition.org/fathom/161

DOI : $10.4000 /$ fathom. 161

ISSN : 2270-6798

Éditeur

Association française sur les études sur Thomas Hardy

Référence électronique

Annie Escuret-Bertrand, "Les romans du Wessex, une esthétique du regard», FATHOM [En ligne],

1 | 2013, mis en ligne le 28 mai 2013, consulté le 26 avril 2019. URL : http://journals.openedition.org/ fathom/161; DOl : 10.4000/fathom.161

Ce document a été généré automatiquement le 26 avril 2019 


\section{Les romans du Wessex, une esthétique du regard}

Le Way-Sex, un éros visuel, de la fem-être à la tombe ou un trajet nommé désir

\section{Annie Escuret-Bertrand}

The novel was, in his own words, "gradually losing artistic form, with a beginning, middle and end, and becoming a spasmodic inventory of items, which has nothing to do with art." (Hardy 1989,

He [...] obliterates the names of the six counties, whose area he traverses in his scenes, under the general appellation of "Wessex" - an old word that became quite popular after the date of Far from the Madding Crowd, where he first introduced

it. So far did he carry this idea of the unity of Wessex that he used to say he had grown to forget the crossing of county boundaries within the ancient kingdom - in this respect being quite unlike the poet Barnes, who was "Dorset" emphatically. (Hardy 1989, 126)

1 Tous les romans ${ }^{1}$ de Thomas Hardy ont un air de famille et cela n'a rien de surprenant si l'on en croit le héros de Proust qui rend compte de la monotonie des œuvres de Vinteuil en affirmant que "les grands littérateurs n'ont jamais fait qu'une seule œuvre, ou plutôt réfracté à travers des milieux divers une même beauté qu'ils apportent au monde" (Proust 375).

2 Si, parmi les nombreux motifs de l'œuvre, nous avons choisi de commencer par une analyse du regard, c'est parce qu'il représente un lieu privilégié des rapports qui se tissent à tous les niveaux. Qui dit "rapport" dit mise en relation, c'est-à-dire accès à la trame du système interactantiel repérable dans le tissu du récit. Certes, il s'agit là d'une 
entrée parmi beaucoup d'autres dans l'espace hardyen, dans ce Wessex au nom pour le moins provocateur ${ }^{2}$ qui nous invite, en quelque sorte, à nous frayer "une route" dans le dédale d'un certain labyrinthe.

\section{La raie et la chevelure}

One evening of late summer, before the present century had reached its thirtieth year, a young man and woman, the latter carrying a child, were approaching the large village of Weydon-Priors on foot. They were plainly but not ill clad, though the thick hoar of dust which had accumulated on their shoes and garments from an obviously long journey lent a disadvantageous shabbiness to their appearance just now. (Hardy 2003b, 3)

3 Cet incipit du Maire de Casterbridge représente, à nos yeux, le début type du récit hardyen. Il suffit, pour s'en convaincre, de lire aussi celui du Retour au pays natal :

Along the road walked an old man [...] Before him stretched the long, laborious road, dry, empty, and white. It was quite open to the heath on each side, and bisected that vast dark surface like the parting-line on a head of black hair, diminishing and bending away on the furthest horizon.

The old man frequently stretched his eyes ahead to gaze over the tract that he had yet to traverse. At length he discerned, a long distance in front of him, a moving spot, which appeared to be a vehicle [...]. (Hardy 1999, 13)

4 Cette longue route blanche qui partage l'étendue d'un noir uniforme est comparée à une raie qui séparerait en deux une chevelure. Cette image figure également dans les premières lignes du manuscrit de The Woodlanders, comme le révèle l'édition critique du roman par Dale Kramer ${ }^{3}$ : "At one place, where a hill is crossed, the largest of the woods shows itself bisected by the highway as a head of thick hair is bisected by the white line of its parting." (Hardy 1981, VI, 5) Cette phrase fut rayée par la suite et remplacée par l'énoncé suivant: "At one place, on the skirts of Blackmoor Vale, where the bold brow of High-Stoy Hill is seen two or three miles ahead, the leaves lie so thick in autumn as to completely bury the track." (Hardy 1981, VI, 5) La suppression intervint sans doute lorsque Hardy réalisa que l'image figurait déjà dans l'incipit de The Return of the Native publié quelques années auparavant. On y trouve cependant les mêmes éléments, à savoir une étendue sombre ("Blackmoor"), un lieu de passage ("the track") et la progression du végétal ("the leaves [...] bury the track"). Si la végétation est la chevelure de la terre, la raie manifeste un passage, une domestication, c'est-à-dire une marque de passage ou d'appropriation. Cette frontière (parting) est active ainsi que l'atteste le suffixe -ing. Elle sépare tout en allant se perdre, de part et d'autre, à l'horizon. Elle oriente, elle donne un sens à l'étendue sombre, tel un signe de craie au tableau noir. C'est elle, enfin, qui permet de mesurer l'écart à franchir ("the tract that he had yet to traverse"). Sans cette route, il n'y aurait qu'un chaos indifférencié. En la traçant, l'homme s'est introduit, s'est dit luimême, comme s'il instaurait l'ordre, la différence, la séparation. Toutefois, cette route est affichée d'emblée comme lieu tragique en ce sens qu'elle n'est ni première, ni unique : “In many portions of its course it overlaid an old vicinal way, which branched from the great Western road of the Romans, the Via Iceniana, or Ikenild Street, hard by." (Hardy 1999, 12) La même métaphore se retrouve dans le poème intitulé "The Roman Road" (Time's Laughingstocks, 1909) :

The Roman Road runs straight and bare

As the pale parting-line in hair

Across the heath. [...] (Hardy 2001, 264) 


$$
\text { déjà là. Cette poussière dégradante ("a disadvantageous shabbiness") est le résultat d'un }
$$
processus : celui de la marche.

6 à-dire de Darwin. Sans le mouvement des jambes et des roues, cette poussière minérale serait restée en l'état. Par conséquent, ce qui circule sur cette route, c'est le mouvement, c'est-à-dire le temps, le temps gestateur. Le processus d'évolution est, d'entrée de jeu, affiché comme dégradant: le terme se nomme Weydon qui peut se lire way/down ou way/done ; chute irréversible, dont le terme (ici le bourg "Weydon Priors") se confond avec la poussière de l'origine. On ne remonte pas le cours du temps et, bien que le trio soit fort jeune, cette poussière va aller s'accumulant jusqu'au moment où elle les réabsorbera tous les trois : l'enfant, puis la femme, et enfin l'homme, dont on nous dit, au dernier chapitre, qu'il a quitté la route pour se fondre dans Egdon Heath, étendue sauvage dont la caractéristique principale est d'être sans route : "[...] that ancient country whose surface never had been stirred to a finger's depth, save by the scratchings of rabbits, since brushed by the feet of the earliest tribes. The tumuli these had left behind, dun and shagged with heather, jutted roundly into the sky from the uplands, as though they were the full breasts of Diana Multimammia supinely extended there." (Hardy 2003b, 318 ; c'est nous qui soulignons) La route linéaire est remplacée, ici, par le tertre funéraire, semihémisphérique, gonflé comme un sein. Cette trouée rectiligne est donc bien, à proprement parler, le lieu de l'écoulement, de la fuite inexorable et irréversible qui mène l'homme à la tombe, tertre ou sein. Nous verrons que le moteur du mouvement qui anime la marche est le désir. C'est lui qui creuse l'écart entre celui qui voit et ce qui est vu, entre la conscience désirante et l'objet désiré. C'est lui qui est à l'origine du déplacement. C'est par lui que tout commence. La sortie de la route représente la mort du désir. Cette route qui se perd à l'horizon sur Egdon Heath représente l'échec de la tentative d'union de la conscience réflexive vaincue par l'enchevêtrement de la végétation, c'est-à-dire, du multiple. En un mot, cette route que nous allons suivre est déjà une flèche couchée qui se perd de bout en bout à l'horizon dans son effort dérisoire de toucher le ciel, là-haut, à la verticale, pareil à cet index gravé par Jude sur une borne milliaire, indiquant la direction de Christ/minster :

A little further on was the summit whence Christminster, or what he had taken for that city, had seemed to be visible. A milestone, now as always, stood at the roadside hard by. Jude drew near it, and felt rather than read the mileage to the city. He remembered that once on his way home he had proudly cut, with his keen new chisel an inscription on the back of that milestone, embodying his aspirations. It had been done in the first week of his apprenticeship, before he had been diverted from his purpose by an unsuitable woman. He wondered if the inscription were legible still, and going to the back of the milestone brushed away the nettles. By the light of a match he could still discern what he had cut so enthusiastically so long ago:

$$
\begin{aligned}
& \text { THITHER --> } \\
& \text { J.F. }
\end{aligned}
$$

The sight of it, unimpaired, within its screen of grass and nettles, lit in his soul a spark of the old fire. Surely his plan should be to move onward through good and ill - to avoid morbid sorrow even though he did see uglinesses in the world? Bene agere et loetari - to do good cheerfully - which he had heard to be the philosophy of one Spinoza, might be his own even now.

He might battle with his evil star, and follow out his original intention. (Hardy 1998b, 73-74)

FATHOM, 1 | 2013 
7 La chevelure de la terre (faite ici d'herbes et d'orties, de bonnes et de mauvaises herbes) a eu raison de la marque du désir "de bien faire". Borne toujours la même et pourtant, à chaque fois, différente! Cette lutte du minéral et du végétal se retrouve dans toute l'œuvre. Certains romans nous disent l'impossible retour "aux sources" tandis que les autres nous parlent de l'impossible restauration, de la chute de l'édifice ou du triomphe du végétal qui engloutit la ruine. Nous voyons s'effondrer des murs, des tours, des châteaux:

Il restera, dans le désert, des pierres, toute une ruine gigantesque délitée lentement par les eaux et le vent, les lentisques, les chèvres, le gel.

Ils se sont séparés, faute de se comprendre, après s'être si bien entendus. Les pierres, maintenant, sont éparses et les ronces traversent les murs. [...] Guerre, fureur, le système est en ruine. Sur ces pierres, nul n'a jamais construit que Babel. (Serres 1982, 199-200)

Ainsi parle Michel Serres de la tour de Babel qu'il oppose à ces tombeaux que sont les “tours achevées" à l'effondrement desquelles les romans du Wessex nous convient dans un univers où sévissent des architectes et des maçons qui passent leur temps à loger les morts tout en oubliant les vivants.

Dans l'autobiographie de Hardy, on trouve à la date du 24 octobre 1892 sa définition de la tragédie: "The best tragedy - highest tragedy in short - is that of the WORTHY encompassed by the INEVITABLE. The tragedies of immoral and worthless people are not of the best." (Hardy 1989, 265) Cela montre le lien entre valeur morale de certains êtres (comme Tess, Jude et Sue) et leur souffrance inique. Si, dans Notre Dame de Paris, Hugo a recours à un sublime de l'horrible en créant le personnage de Quasimodo, Hardy fait de même avec le personnage allégorique de Little Father Time et, en choisissant d'humbles mortels pour héros tragiques, il opère une démocratisation du sublime tragique : chez lui, la noblesse est purement morale (ainsi que le souligne le sous-titre de Tess of the d'Urbervilles: The Story of a Pure Woman). Hardy n'est pas marxiste mais Jude et Sue sont tués par la société qui ne leur donne pas leur "place".

10 L'histoire de Jude retrace sa lutte contre les différentes formes de "loi" : la loi de la nature à Marygreen (dont le représentant est Arabella Donn), l'injustice sociale à Christminster, la loi de l'Église à Melchester, la loi qu'il s'est donné à lui-même à Shaston et à Aldbrickham et ainsi de suite. À l'époque moderne, la vision tragique se double d'une vision ironique et absurde car on assiste à un effondrement des superstructures mythiques chez Hardy, Camus, Ionesco ou Beckett. La mort a toujours le dernier mot dans les romans de Hardy car, chez lui, les forces sociales ne sont que des mécaniques aveugles qui détruisent tout sur leur passage. Hardy ne croit plus en l'homme et voilà pourquoi Jude the Obscure se termine sur des paroles blasphématoires de décréation. Jude aurait préféré ne jamais voir le jour :

'Let the day perish wherein I was born and the night in which it was said, there is a man child conceived.'

('Hurrah!') [...] (Hardy 1998b, 403 ; c'est l'auteur qui souligne)

11 Confronté à l'impossibilité de venir se "ressourcer" aux lieux familiers, le héros "quitte la route". Dès cet instant, il se trouve réduit à un point minuscule qui se confond avec le paysage. Songeons à Tess dans les champs de Flintcomb Ash, à Fanny Robin perdue dans la neige, à Clym devenu coupeur d'ajoncs! Ne plus être sur la route signifie donc la fusion de l'individu dans la masse, de l'un dans le multiple, de la pierre dans les ronces. Cette fusion n'intervient qu'au dénouement. Tel est, en gros, le schéma dynamique que nous 
allons retrouver dans notre étude des rapports visuels qui constituent cette voie du Désir nommée Wessex.

Le voyageur qui parcourt inlassablement le chemin du désir est, au départ, aussi seul que l'homme sur la route blanche. Comme lui, il ne tarde guère à apercevoir une autre créature qui circule, solitaire. Ces deux individus sont les deux pôles du récit, de l'échange. Un individu seul, par définition, n'a pas d'histoire. S'il n'y a pas d'histoire, il n'y a pas de discours narratif et réciproquement. Ces deux pôles correspondent à deux positions spatiales qui délimitent un segment, une distance, un écart. C'est donc l'écart qui est constitutif du drame visuel inaugural car certains veulent le réduire tandis que d'autres préfèrent le creuser. En effet, si l'espace du Wessex n'est là que pour être traversé de regards, la place occupée par les protagonistes est subordonnée à leurs rapports visuels car la logique exige que l'on sache comment, par exemple, Gabriel Oak peut épier Bathsheba sans être vu, ou bien, comment Troy fascine la jeune fille tout en étant lui-même fasciné. L'œil de l'architecte se retrouve, bien entendu, dans 1'attention accordée aux oppositions. Ceci explique, sans doute, les nombreuses descriptions d'édifices qui jalonnent ce Wessex, de Desperate Remedies à Jude the Obscure. Hardy avoua un jour à Sir Alfred Richardson (lui-même architecte) : "Architecture taught me to place one thing on the other [...] and I began to select views [...] and localities - it made a great impression on me" (Beatty 32). Dans la biographie, on peut lire : "He had fortified himself in his opinion by thinking of the analogy of architecture, between which art and that of poetry he had discovered, to use his own words, that there existed a close and curious parallel, both arts, unlike some others having to carry a rational content inside their artistic form." (Hardy 1989, 323)

Il n'est donc pas surprenant de voir la perspective (ou la focalisation) jouer un grand rôle dans cette poétique de l'espace. Dès le premier roman, on remarque ce souci de "placer" les acteurs. L'héroïne, Cytherea Graye, aperçoit son père (qui est architecte!) sur un échafaudage. Auparavant, le narrateur a pris soin de situer la jeune fille : elle regarde par la fenêtre de l'Hôtel de Ville d'où elle a une vue plongeante sur l'église que son père est en train de restaurer: "The picture thus presented to a spectator in the Town Hall was curious and striking. It was an illuminated miniature, framed in by the dark margin of the window, the keen-edged shadiness of which emphasized by contrast the softness of the objects enclosed." (Hardy 1998a,14) Dans The Mayor of Casterbridge, Henchard épie Farfrae et Elizabeth-Jane, du haut du fort préhistorique nommé "Mai Dun". Sa situation physique privilégiée lui permet d'observer les alentours sans être vu : "A quarter of a mile from the highway was a pre-historic fort, of huge dimensions and many ramparts, within or upon whose enclosures a human being, as seen from the road, was but an insignificant speck. Hither Henchard often resorted, glass in hand, and scanned the hedgeless Via [...] to a distance of two or three miles [...]." (Hardy 2003b, 304) Eustacia Vye, quant à elle, utilise une lunette pour se jouer de la distance au cours de ses promenades sur la Lande.

Il en va des acteurs comme du narrateur. Sans l'œil, il ne pourrait pas remplir son office ainsi qu'en témoigne le premier chapitre du Retour au Pays Natal. La gageure consiste à vouloir dire la lande avant l'arrivée de l'homme, comme si l'on pouvait focaliser sans l'œil. Le nombre de notations d'ordre visuel est tel que le lecteur comprend qu'il s'agit là d'un pari impossible: "Looking upwards, a furze-cutter would have been inclined to continue work" ; (Hardy 1999, 9 ; c'est nous qui soulignons); "when night showed itself an apparent tendency to gravitate together could be perceived in its shades and the scene" (Hardy 1999, 10); "It was a spot which returned upon the memory of those who loved it 
with an aspect of peculiar and kindly congruity" (Hardy 1999, 10); “To recline on a stump of thorn in the central valley of Egdon, between afternoon and night, as now, where the eye could reach nothing of the world outside the summits and shoulders of heathland which filled the whole circumference of its glance" (Hardy 1999, 12; c'est nous qui soulignons). Puisque le narrateur est censé ne pas être là, il opère donc par le truchement de spectateurs hypothétiques.

Le titre du chapitre I est : "A Face on Which Time makes but Little Impression" tandis que le second chapitre s'intitule: "Humanity appears upon the Scene, Hand in Hand with Trouble". L'apparition de l'homme est synonyme de "trouble", c'est-à-dire de bruit et de fureur qui vont se déchaîner, comme si au tumulte des choses se joignait la haine des hommes. On reconnaît là cette "noise" dont nous parle Michel Serres dans Genèse : "Noise, intermittence et turbulence, querelle et bruit, cette noise marine est la rumeur originaire, elle est la haine originelle. Nous l'entendons en haute mer." (Serres 1982, 33) Cette belle querelleuse ne saurait être que marine à en croire cet autre roman de Hardy ( Far from the Madding (rowd) où le fauteur de troubles se nomme le capitaine Troy, écho d'une autre querelle maritime célèbre. Troy disparaît dans la mer pour ressurgir un beau soir chez Boldwood qui l'abat d'un coup de fusil. Non, il n'y a pas de lieu à l'abri du bruit et de la fureur. Dans The Return of the Native, on voit donc s'opérer insensiblement un glissement de l'œil à l'oreille du premier au second chapitre. Les deux voyageurs se rejoignent et les seuls bruits mentionnés sont ceux du vent, des roues et de l'attelage : "The silence conveyed to neither any sense of awkwardness; in these lonely places wayfarers, after a first greeting, frequently plod on for miles without speech." (Hardy 1999, 14-15) Ce qui va rompre le silence, c'est le bruit de la conversation qui s'engage parce que l'homme au rouge ne cesse d'aller jeter "un coup d'œil" au "contenu" de sa roulotte :

Possibly these two might not have spoken again till their parting, had it not been, for the reddleman's visits to his van. When he returned from his fifth time of looking in the old man said, "You have something inside there besides your load?"

"Yes."

"Somebody who wants looking after."

"Yes" [...].

"You have a child there, my man?"

"No, sir, I have a woman." (Hardy 1999, 15)

La jeune femme en question n'est autre que Thomasin dont Venn est amoureux. Il se contente de la regarder pendant qu'elle dort! Son plaisir manifeste excite la curiosité de l'autre voyageur qui aimerait bien en faire autant. Venn, toutefois, ne le laisse pas approcher de la voiture. En d'autres termes, nous venons d'assister à la genèse de l'histoire par le truchement d'un acte de regard qui précède l'échange verbal. Sans l'autre voyageur, nous n'aurions pas pu pénétrer ainsi au cœur de la relation dans la mesure où ce "voyageur anonyme" qui aimerait bien voir lui aussi est le lecteur ... frustré !

17 Le discours de l'instance énonciatrice est le canal visuel, auditif et "bruyant" qui négocie la noise ... pour un temps. En effet, le narrateur (ou énonciateur) est une sorte de Protée qui se fait bouche, oreille et œil. Ces organes canalisent toute l'information et la parasitent souvent, comme il se doit. Cependant, si l'on neutralise pour un temps les 
éléments parasites, on peut repérer un certain nombre d'invariants constitutifs de la scène originelle du drame visuel.

Parmi ces éléments, on remarque d'abord le rôle joué par la topographie. Bien entendu, le problème n'est pas de savoir si Endelstow est une réplique fidèle ou non de St. Juliot où Hardy fit la connaissance de sa première femme ou bien si la tour qui sert d'observatoire à Swithin St. Cleeve dans Two on a Tower est vraiment celle de Charborough Park, mais de constater les rapports particuliers des habitats entre eux ${ }^{4}$. Il faut d'abord remarquer que l'action se déroule dans un cercle inexorable de lieux. On nous dit, par exemple, que Little Hintock, dans The Woodlanders, "was one of those sequestered spots outside the gates of the world where may usually be found more meditation than action, and more listlessness than meditation; where reasoning proceeds on narrow premisses, and results in inferences wildly imaginative; yet where, from time to time, no less than in other places, dramas of a grandeur and unity truly Sophoclean are enacted in the real, by virtue of the concentrated passions and closely-knit interdependence of the lives therein." (Hardy 1998d, 8; c'est nous qui soulignons) La passion bien connue de Hardy pour les tragiques grecs se retrouve dans cette soumission des personnages au lieu ainsi que dans ce choix délibéré d'une unité de lieu : la Lande dans The Return of the Native, les bois dans The Woodlanders, ou la ville de Casterbridge dans The Mayor of Casterbridge. Pensons à ces petits villages comme Carriford, Mellstock, Sleeping-Green, Endelstow, Little Hintock, Marygreen, Marlott, à ces belles demeures situées à l'écart, cachées au fond d'un parc : Knapwater House où vit Miss Aldclyffe, Welland House, lieu de résidence de Lady Constantine, le château des De Stancy, le domaine de Mrs Charmond et bien d'autres encore. Les habitants ont la possibilité de se rendre d'un village à un autre, d'une ville à une autre, d'une habitation à une autre. On peut même dire qu'on se déplace beaucoup dans les romans du Wessex mais la réalité tragique est partout. Un départ pour le Brésil, l'Italie ou les Indes ne règle rien. Il n'y a pas deux sphères dont l'une (le Wessex) serait tragique et l'autre pas. En d'autres termes, il n'y a qu'une seule réalité humaine (The Way of Sex) dont la quotidienneté est faite d'événements et non d'exceptions à une "règle". Cette négation ${ }^{5}$ de l'existence d'une sphère autre que la réalité tragique se retrouvera dans la cosmisation des rapports qui abolit toute différence entre forces cosmiques et forces humaines. Au niveau humain, cette négation apparaît aussi dans la représentation des rapports entre les sexes. La femme ne constitue pas un "ailleurs" pour l'homme et réciproquement. La réalité tragique concerne tout le monde, abolissant la frontière entre les sexes, le dedans et le dehors, le proche et le lointain, l'étranger et l'autochtone, le visible et l'invisible.

Le premier choc entre deux monades est en général fortuit et c'est lui qui est tragique en ce sens qu'il est imprévisible. On nous dit, par exemple, que Jude et Arabella n'ont au départ aucun point commun "except [...] locality" (Hardy 1998b, 44). Si la première rencontre est accidentelle, les déplacements qui suivent, en revanche, sont régis par ce premier regard. C'est par hasard que Lady Constantine découvre Swithin dans la tour. Si Jude n'avait pas un soir changé d'itinéraire, Arabella Donn ne se serait jamais trouvée sur son chemin. C'est par hasard que les yeux de Jude se posent sur la photographie de Sue. C'est par hasard que Gabriel Oak rencontre Bathsheba la première fois. C'est en chantant avec le chœur de Mellstock dans les rues que Dick voit un soir Fancy à sa fenêtre. Somerset fait la connaissance de Paula après l'avoir vue se dérober à la cérémonie du baptême dont il a été le témoin involontaire. Ce premier regard est lourd de conséquences car il est invariablement suivi de multiples rapports visuels. Bien souvent 
un déplacement, un incident, une nouvelle rencontre ne sont que l'occasion d'un autre regard. Les exemples abondent; pensons, entre autres, à la rencontre des héros de Jude the Obscure lors de la grande foire agricole de Stoke-Barehills : elle permet à Arabella de revoir Jude, de l'épier tandis qu'il se promène avec Sue, d'observer la façon dont ils se regardent. Sa jalousie éclate car elle se rend parfaitement compte que Jude ne l'a jamais regardée ainsi. "You never see anything", dit-elle à Cartlett que les deux amoureux n'intéressent guère (Hardy 1998b, 292). La trame de l'œuvre est donc tissée de regards qui sont à proprement parler constitutifs de l'espace way/sex, lieu de l'éros visuel.

La première rencontre ou le premier regard accidentel est la scène centrale autour de laquelle le drame va s'organiser. Ce face-à-face initial prend en général la forme d'une fascination. L'objet fascinant est soit un homme, soit une femme: l'appartenance à un sexe plutôt qu'à l'autre ne détermine donc pas à l'avance la victime ou le bourreau. Toutefois, chaque sexe possède des armes qui lui sont propres : la coquetterie pour les femmes, l'uniforme ou la musique pour les hommes. On peut d'abord voir comment les femmes s'y prennent pour attirer les solitaires du sexe fort qui passent dans leur champ visuel. Les racines de la coquetterie sont tellement profondes qu'on peut aller jusqu'à dire que la femme hardyenne ne vit qu'en fonction du regard d'autrui. Elle s'acharne à plaire dans un souci perpétuel de l'opinion des autres. Elle a sans cesse conscience du fait qu'elle est regardée ou bien qu'elle peut être vue. Dans Under the Greenwood Tree, Mr Dewy nous offre une description saisissante de ce phénomène : "Now Dick, this is how a maid is", ditil à son fils en guise d'avertissement. "She'll swear she's dying for thee, and she is dying for thee, and she will die for thee ; but she'll fling a look over t'other shoulder at another young feller, though never leaving off dying for thee just the same !" (Hardy 2004, 87) La coquetterie a toujours le dernier mot, même aux heures les plus graves. Hardy nous en donne de multiples exemples dans ses romans et dans ses poèmes. Dans le poème "The Pink Frock" (Moments of Vision, 1917), nous voyons une belle reprocher à son amant d'être mort le jour où elle devait étrenner une jolie robe rose :

'O my pretty pink frock

I sha'n't be able to wear it

Why is he dying just now?

I hardly can bear it!

'He might have contrived to live on

But they say there's no hope whatever

And must I shut myself up, And go out never?

'O my pretty pink frock!

Puff-sleeved and accordion-pleated

He might have passed in July,

And not so cheated!' (Hardy 2001, 472)

Dans Desperate Remedies, le narrateur declare: "Few women, if taxed with having an admirer, are so free from vanity as to deny the impeachment, even if it is utterly untrue." (Hardy 1998a, 121) Dès les premières œuvres, l'accent est mis sur ce travers qui constitue l'essence de la femme : "Woman's ruling passion - to fascinate and influence those more powerful than she" (Hardy 1998c, 192). Une femme se préoccupe de son apparence jusqu'à son dernier souffle : "Catch a woman napping if you can, even when she's asleep or half dead" (Hardy 1998a, 38). Rien ne vient interrompre l'exercice de la coquetterie dont le but est à proprement parler de réduire l'écart qui sépare les deux pôles conformément à la loi de l'attraction : écoutons Sue déclarer avec la franchise qui la caractérise, "[...] how much I feel that I shouldn't have been provided with attractiveness unless it were meant to be 
exercised!" (Hardy 1998b, 204) La coquette se constitue donc en objet fascinant et elle éblouit ses victimes qui se laissent asservir par une apparence éclatante.

Parmi les héros victimes de la fascination, Jude Fawley et William Boldwood semblent être les plus pathétiques. Dans le cas de la première rencontre de Jude avec Arabella, tout commence par un seul échange de regards. On se souvient que Jude rentrait chez lui ce soir-là, aveugle au spectacle qui l'entourait, sourd aux appels qui provenaient de l'autre côté de la haie où se tenaient Arabella et ses deux amies. Bathsheba utilise "a valentine" pour attirer le regard de Boldwood; Arabella lance un sexe de verrat pour capter celui de Jude et la suite est facile à deviner: "The unvoiced call of woman to man, which was uttered very distinctly by Arabella's personality, held Jude to the spot against his intention - almost against his will, and in a way new to his experience." (Hardy 1998b, 40 ; c'est nous qui soulignons). Dans la mesure où un regard exprime une relation intentionnelle avec autrui on peut facilement deviner les intentions d'un personnage qui regarde en n'ayant pas conscience d'être lui-même observé. Le regard que Jude porte sur les bras et les seins d'Arabella est des plus éloquents. Cette rencontre n'est cependant pas un phénomène exceptionnel. Jude fait simplement connaissance avec une force qualifiée de "the unvoiced call of woman to man". Cette force "qui ne parle pas" affecte tous les hommes en général et Jude ne fait ici que répéter ce que tous les hommes ont fait avant lui. On reconnaît là, bien entendu, le caractère tragique de l'événement qui est toujours fondamentalement la répétition d'un acte dont le tragique tient aussi au fait qu'il est "unvoiced", silencieux, muet. Il s'agit là de la manifestation d'une force que la parole n'exprime pas mais qui ne saurait surprendre ces hommes habitués à cheminer des heures, côte à côte, sans parler.

Les deux héros se rejoignent au milieu du petit pont qui enjambe le cours d'eau et Jude rend à Arabella le sexe de verrat. En ramassant puis en lui rendant son bien, il relève pour ainsi dire le défi qu'elle lui a lancé. La relation essentielle est contenue en germe dans cette première rencontre : Arabella est d'ores et déjà associée aux pourceaux, symbole des tendances obscures sous toutes leurs formes. Le sexe de verrat tombe aux pieds de Jude ; or, à ce moment précis, il se tenait immobile, fixant le sol, comme si son avenir s'y trouvait projeté. Il se voyait en rêve faisant de brillantes études à Christminster. Le sexe de verrat est l'intrusion de la sensualité dans la vie du héros et le mirage se dissipe devant cet assaut du réel. Lui qui allait se jucher sur le toit de la Maison Brune afin de contempler les lumières de Christminster dans le lointain se laisse prendre au piège par la brune Arabella. L'éblouissement provoque une sourde effervescence en Jude. Toutefois, il ne tarde guère à prendre conscience du fait que ce bouleversement n'a pas simplement une cause externe. En un mot, il "se" reconnaît dans ce qui lui arrive.

Cette reconnaissance est fondamentale en ce sens qu'elle est constitutive du héros tragique; elle abolit toute distinction entre cause et conséquence, innocent et coupable, bourreau et victime. Tess devient Alec (en le tuant), Manston-Eunice, Jude-Arabella. Cette fascination brutale n'est donc pas l'intrusion d'un élément étranger à Jude. Il y a en lui une force dont il ne soupçonnait pas l'existence et qu'il découvre brutalement. À en croire le narrateur, ces lignes de force visuelles qui relient les deux protagonistes à travers l'espace où, un moment auparavant, ils se trouvaient séparés, sont les mêmes que celles qui régissent les phénomènes électromagnétiques. Hardy écrivit Jude l'obscur entre 1890 et 1894. En 1895, Pierre Curie opère, comme par hasard, une distinction entre paramagnétisme et diamagnétisme et met en évidence le passage du ferromagnétisme au paramagnétisme par élévation de température. Les phénomènes ferromagnétiques sont 
fort intéressants car leur fonctionnement ressemble curieusement à celui des acteurs hardyens. L'Encyclopoedia Universalis nous dit ceci :

L'hystérésis

Lorsqu'un corps ferromagnétique à l'état vierge, c'est-à-dire qui n'a jamais été aimanté, est soumis à un champ magnétique croissant, son aimantation $\mathrm{M}$ commence par croître, selon la courbe de première aimantation. [...] Lorsque le champ prend des valeurs élevées, $M$ tend vers une limite $\mathrm{M}_{\mathrm{s}}$ appelée aimantation à saturation. [...]

[L]orsqu'on soumet l'échantillon à un champ alternatif lentement décroissant [...] l'aimantation décrit une série de cycles partiels de dimensions décroissantes qui s'emboîtent les uns à l'intérieur des autres pour aboutir à une aimantation nulle dans un champ nul. L'échantillon a été désaimanté [...]. (Encyclopédie thématique 2408-2410)

On ne peut s'empêcher de rêver devant cette "rencontre" entre science et littérature! Bien entendu, l'intérêt n'est pas de savoir si Hardy s'intéressait ou non à l'hystérésis mais d'observer qu'il dit l'hystérésis dans la mesure où les rapports entre Jude et Arabella peuvent être assimilés aux phénomènes d'aimantation. "Aimant" veut dire "qui aime", qui attire. Toutefois, la masse qui aimante n'attire que les corps en mesure de répondre au "signal". Arabella est la terre susceptible d'être charruée. On la voit couver un œuf qu'elle a niché entre ses seins. Elle est donc, au départ, la source de chaleur ou d'énergie. C'est elle qui aimante et Jude atteint rapidement la phase de saturation. Le champ alternatif qui le "désaimante" est l'autre visage d'Arabella, celui de la mégère qui a remplacé la séductrice. L'élément ferromagnétique qui permet à Jude de répondre à l'aimantation n'est autre que sa sensualité. À la fin, lorsqu'il est étendu sur son lit, raide comme une flèche ("straight as an arrow" [Hardy 1998b, 407]), Arabella comprend que Jude ne pourra plus être son "soc" et part sur le champ avec Vilbert.

La seconde aimantation s'appelle Sue, prénom que Richard Phillotson prononce "Soo" (Hardy 1998b, 220) qui est, nous dit Norman Holland, "the traditional call for pigs, which symbolizes his sexual attitude" (Holland 56). Phillotson est aimanté par Sue mais l'inverse est vrai aussi : Sue retourne à Phillotson, comme poussée par une force "inconnue" qui se trouve en elle. Ces changements brusques de direction de l'aiguille aimantée (Sue) qui tantôt pointe vers Jude, tantôt vers Phillotson, est le signe d'un dérèglement qui renvoie à une perte du sens (de l'orientation), c'est-à-dire, fondamentalement au non-être. En d'autres termes, ce prétendu "masochisme" signifie en fait la dénaturation, l'état de mort qui caractérise l'existence humaine. Ce jeu de l'aiguille folle se retrouve dans la métaphore de la coquette ou du Don Juan. En effet, si Jude est de toute évidence victime de la coquetterie d'Arabella, de ses "fossettes artificielles" et de ses faux cheveux, il est aussi victime de celle de sa cousine. Sue est à ce propos encore une fois remarquable par sa franchise. Elle avoue à Jude : "At first, I did not love you Jude ; that I own. When I first knew you, I merely wanted you to love me. I did not exactly flirt with you; but that inborn craving which undermines some women's morals almost more than unbridled passion - the craving to attract and captivate, regardless of the injury it may do the man - was in me" (Hardy 1998b, 353). Ainsi, la femme se constitue en objet fascinant et l'homme, à moins qu'il ne soit "de bois", est pris, sur le champ, au piège de l'aimantation.

Fancy Day paraît à sa fenêtre et Dick Dewy, à l'instant même, lui appartient. De façon très symbolique, elle est auréolée de lumière tandis que Dick se tient en bas, dans l'obscurité, parmi les autres musiciens. Fancy ou la "chimère" dont le nom (Day) évoque l'éclat aveuglant du jour. Tout commence par cette fulguration instantanée : Fancy est vue par 
Dick et celui-ci est ébloui par Fancy et l'on devine sans peine qui sera victime. Il suffit, pour s'en convaincre, de relire le chapitre cinq de Under the Greenwood Tree intitulé "The Listeners". En fait de "listeners", l'on est plutôt en présence d'un groupe de "watchers" ou de "peepers". Les chanteurs de Mellstock sont sous les fenêtres de la jeune institutrice et ils s'éloignent pour s'apercevoir, quelques minutes plus tard, que Dick ne se trouve plus parmi eux. Ils reviennent sur leurs pas et le découvrent sous la fenêtre, au même endroit. À partir de ce moment, Dick n'a plus qu'une idée en tête : épouser Fancy. Ses tribulations ne font que commencer. Fancy est une sorte de virus qui transforme Dick en un autre que lui-même. Il devient sa "chose". Cette métamorphose de l'autre en "même" est un bel exemple de contamination par la fem/être. Nous le voyons faire des efforts pathétiques afin de trouver grâce aux yeux de cette coquette. Fancy ne se contente pas d'éblouir un seul homme et il lui est très difficile de renoncer aux hommages, alors même qu'elle prétend aimer l'une de ses victimes. Cela vient de ce qu'aimer pour une femme compte bien moins à ses yeux qu'être aimée. Son instinct de possession la pousse à multiplier le nombre de ses conquêtes, tel un pouvoir magnétique qui s'exercerait parce qu'il est magnétique. Cette force est elle-même contingente tandis que ce qu'elle provoque constitue la chaîne de nécessités fatales.

La coquetterie de la femme lui tient lieu d'être car elle ne vit qu'en fonction de la possibilité permanente d'être vue par autrui. Elle prend un plaisir indicible à s'offrir éblouissante à la vue de l'univers. Si un regard refuse de se laisser éblouir, elle n'aura de cesse qu'elle ne l'ait subjugué. C'est là le drame de William Boldwood. Nous nous souvenons de la première apparition de Bathsheba Everdene, l'héroïne de Far from the Madding Crowd, au marché aux blés à Casterbridge. Tandis que tous les regards convergeaient vers elle, un seul homme eut l'audace de lui refuser cet hommage. Il suffit de relire le chapitre XII intitulé "Farmers - A Rule - An Exception" pour être convaincu de l'importance de l'offense :

The numerous evidences of her power to attract were only thrown into greater relief by a marked exception. Women seem to have eyes in their ribbons for such matters as these. Bathsheba, without looking within a right angle of him, was conscious of a black sheep among the flock.

[...] Bathsheba was convinced that this unmoved person was not a married man. (Hardy 2003a, 81)

Bathsheba apprend que le bel indifférent est un célibataire endurci du nom de William Boldwood. "Never was such a hopeless man for a woman! He's been courted by sixes and sevens - all the girls gentle and simple for miles round have tried him." (Hardy 2003a, 67) Telle est la description qu'en donne Mrs Coggan. Bathsheba va s'efforcer de réussir là où d'autres ont échoué en ayant recours à la ruse. Lorsqu'il reçoit le jour de la SaintValentin le billet portant les mots “Épousez-moi” qu'elle lui a envoyé par plaisanterie afin de se venger de son indifférence, le superbe isolement prend fin. Cet homme insensible aux charmes féminins est intrigué, puis troublé. Lorsqu'il découvre l'auteur du billet, il cherchera à la voir et se damnera en même temps. Le narrateur rend compte de la transformation brutale qui s'opère en lui 1e jour où il voit Bathsheba de la façon suivante : "Adam had awakened from his deep sleep, and behold, there was Eve" (Hardy 2003a, 102). Avant cet incident, Boldwood regardait les femmes sans les voir! Bathsheba constate que sa ruse a réussi : "All this time Bathsheba was conscious of having broken into that dignified stronghold at last. His eyes, she knew, were following her everywhere." (Hardy 2003a, 103) Lorsque Boldwood lui déclare son amour, elle réalise pleinement combien son jeu innocent était coupable. Le piège se referme sur la 
malheureuse victime car, plus Bathsheba s'éloigne et plus Boldwood la désire. Dans l'exacte mesure où il est fascinant, l'objet convoité ne peut être possédé. En le fascinant, Bathsheba est responsable de sa perte. Cependant, Boldwood ne meurt pas pour elle : il meurt pour une promesse qu'elle n'a jamais donnée. Le billet anonyme scellé du cachet portant l'inscription: "Marry Me" n'engageait pas l'expéditeur. La forteresse de Boldwood n'était pas un refuge imprenable puisque la poste se charge d'acheminer la femme (lettre) qu'il avait refoulée. S'il a pu rêver un instant de s'emparer de la jeune fille, les rôles ont tôt fait de se trouver inversés : le voici soudain passif et paralysé. Il renonce à sa volonté propre pour se laisser dominer par l'appel de l'inaccessible.

Ce gentilhomme-campagnard modèle néglige sa ferme, ses récoltes: "A few months earlier Boldwood's forgetting his husbandry would have been as preposterous an idea as a sailor forgetting he was in a ship. Oak was just thinking that whatever he himself might have suffered from Bathsheba's marriage, here was a man who had suffered more" (Hardy 2003a, 224). La mort sera au bout de la nuit puisque Boldwood tue le mari de Bathsheba et finit ses jours dans un asile. L'ombre de la cellule n'est que le prolongement de l'obscurité, de l'aveuglement produit par l'éblouissement initial. Cette fin tragique est contenue en germe dans les premières scènes où apparaît Boldwood lorsqu'il contemple fixement le billet envoyé par Bathsheba : "the large red seal became as a blot of blood on the retina of his eye" (Hardy 2003a, 87). Cette phrase oblige le lecteur à prendre conscience du trouble anormal de Boldwood. À force de fixer le cachet scellant l'enveloppe $\mathrm{du}$ message, Boldwood s'aveugle physiquement tout en s'aliénant psychiquement. Cette tache rouge qui marque le commencement de sa passion annonce la tache de sang que laissera la balle qu'il tirera sur Troy et qui, du même coup, mettra un terme à son égarement. Ce billet l'obsède jour et nuit et il ne parvient plus à faire la part du rêve et de la réalité.

31 Lorsque la femme fait figure de victime, le bourreau a pour nom Francis Troy, Alec d'Urbervilles, Edred Fitzpiers, Henry Knight, Angel Clare ou Clym Yeobright. Troy, Alec et Fitzpiers font irruption dans la vie de Bathsheba, Tess et Grace. La première rencontre de Troy avec Bathsheba ou d'Aeneas Manston avec Cytherea est une prise de possession violente et soudaine. Leurs regards se croisent par hasard et une tension s'installe : c'est le choc de deux destinées qui se révèlent brusquement l'une à l'autre et, pourrait-on dire, l'une par l'autre. Ce premier face-à-face n'a pas lieu en plein jour mais la nuit, ou bien sous un ciel d'orage. La première rencontre de Troy avec Bathsheba préfigure leurs rapports à venir. Ils se heurtent dans l'obscurité : "a figure was apparently on the point of gliding past her when something tugged at her skirt and pinned it forcibly to the ground. The instantaneous check nearly threw Bathsheba off her balance. In recovering she struck against warm clothes and buttons." (Hardy 2003a, 141) L'éperon lacère l'étoffe délicate de la robe de Bathsheba. Le dialogue qui s'élève alors dans l'obscurité traduit bien cette reconnaissance originelle du rôle dévolu par la nature à chacun des deux protagonistes :

"A rum start, upon my soul!" said a masculine voice, a foot or so above her head.

"Have I hurt you, mate?"

"No", said Bathsheba, attempting to shrink away.

"We have got hitched together somehow, I think."

"Yes."

"Are you a woman?"

"Yes."

"A lady, I should have said." 
"It does not matter."

"I am a man."

“Oh!” (Hardy 2003a, 141) nuit annonce leur relation essentielle. Le spectacle que Bathsheba contemple n'a qu'un lointain rapport avec le réel car la lanterne, avec ses jeux d'ombres et de lumière, ne dissipe pas vraiment les ténèbres. À cette vision déformée s'ajoute l'éclat aveuglant de l'uniforme rutilant de Troy. La lanterne, nous dit-on, "radiated upwards into their faces, and sent over half the plantation gigantic shadows of both man and woman, each dusky shape becoming distorted and mangled upon the tree-trunks till it wasted to nothing." (Hardy 2003a, 142) Le lecteur constate que, dès leur premier rapport visuel, les deux héros sont dupes des apparences. La couleur flamboyante de l'uniforme tranche d'une manière désagréable sur le cadre naturel où se déroule la scène. Les ravages que Troy va causer dans l'existence de Bathsheba sont contenus en germe dans cette rencontre inaugurale. En effet, ses disparitions soudaines, sa façon de se travestir, sa cruauté le font souvent ressembler à un personnage démoniaque. La jeune femme est donc doublement captive puisqu'elle est fascinée par le bel uniforme et par le regard de cet inconnu dont l'éperon la retient prisonnière. Ce regard qu'ils échangent dans la nuit a véritablement la valeur d'un événement premier. C'est parce qu'ils se sont vus que chacun ne pourra plus oublier le visage de l'autre: Bathsheba oublie l'infortuné Boldwood sur le champ et Troy est lui aussi fasciné par le visage de Bathsheba puisqu'il ne songe plus du tout à Fanny. Les scènes suivantes sont le prolongement naturel de ce premier éblouissement réciproque. L'habit militaire est souvent associé à la mort, au danger, à l'orgueil, au mal dans les romans du Wessex. Miss Aldclyffe, Anne Garland, la mère d'Eustacia sont victimes de l'uniforme.

La fulguration n'est là que pour rappeler les vertus destructrices de l'incandescence originelle. Cette assimilation de la personne à un orage par le biais d'un isomorphisme regard-lumière est typique d'une conception romantique du débordement des passions incontrôlables. Les héros inflammables tels Wildeve, Boldwood ou Eustacia meurent foudroyés tandis que Gabriel Oak est celui qui installe un paratonnerre de fortune sur les meules de Bathsheba. Rien d'étonnant à ce que ce soit lui le vainqueur, lui qui sait canaliser l'électricité magnétique. Si l'homme est un corps ferromagnétique (ainsi que l'attestent les éperons et l'épée de Troy), l'attirance qu'il subit cesse dès qu'il est "désaimanté" ou bien dès qu'une masse magnétique plus forte l'attire vers elle. C'est ainsi que nous pouvons rendre compte du comportement de "l'homme à femmes". En effet, le mariage ne suffit pas à mettre l'époux à l'abri de la tentation, bien au contraire, car la mort du désir est la loi de toute possession. Troy, Aeneas, Fitzpiers sont victimes d'éblouissements successifs. Troy délaisse Fanny Robin, Aeneas sa femme Eunice, Fitzpiers sa jeune épouse Grace.

Le drame de la fascination comporte un vainqueur et un vaincu mais le bourreau fait le plus souvent figure de victime par la suite. Troy, Alec, Aeneas sont plus à plaindre que leur victime et leur mort brutale est l'illustration parfaite du danger qui guette le séducteur hardyen. Certains amants inconstants connaissent toutefois un sort plus clément. Dans The Trumpet-Major, Bob Loveday, l'un des deux fils du meunier, est lui aussi victime d'aimantations successives. Dès qu'il s'éloigne d'Anne Garland, il la trompe. Elle ne règne sur lui que par sa présence. Quand son regard se détourne de l'objet aimé, une nouvelle aimantation se produit mais provoquée par un autre astre, nommé Mathilda ou

FATHOM, 1 | 2013 
Caroline. Comme l'effet s'évanouit quand le regard se détourne de l'être éclatant, rien ne perpétue l'événement magique, à moins qu'il ne reçoive la durée par un décret de la raison ou de la volonté. Cet acte de volonté s'exprimera sous la forme du serment ainsi que nous l'explique Jean Starobinski : "Le serment décide que la femme aimée est unique, et il fonde de la sorte un avenir continu. Soumise à la loi du regard, la vie risque de se disperser en une multiplicité de ravissements fugitifs; il faut lui imposer la loi de la parole, qui lie l'être à lui-même et le contraint à se rassembler tout entier dans sa constance. Qui a donné sa parole ne peut désormais être infidèle qu'au prix d'un parjure déshonorant. Et si, contre toutes les contraintes et toutes les séductions extérieures, il parvient à tenir sa promesse, le voici devenu glorieux, et offert à l'admiration universelle." (Starobinski 38-39) Ces remarques de Jean Starobinski à propos du héros cornélien peuvent s'appliquer à la seconde catégorie de héros masculins représentée par Bob Loveday par exemple. Le docteur Fitzpiers et le fils du meunier sont des séducteurs mais ils échappent au sort habituellement réservé à leurs semblables.

En fait, si l'on examine attentivement les héros masculins des principaux romans du Wessex, l'on s'aperçoit que leur attitude visuelle détermine toute leur existence. Il n'y a que trois types d'hommes que l'on pourrait définir de la façon suivante : les victimes de l'éblouissement, les voyeurs et les aveugles.

- John Loveday, Edward Springrove, Stephen Smith, Gabriel Oak, Christopher Julian, Diggory Venn, William Dare, Lord Mountclere, Captain de Stancy, Giles Winterborne, Jocelyn Pierston font partie des voyeurs.

- Bob Loveday, Manston, Wildeve, Fitzpiers, Alec, Troy, Boldwood et Jude sont victimes d'un ou de plusieurs éblouissements comme nous venons de le constater.

- Swithin St. Cleeve, Henry Knight, Angel Clare sont aveugles ; Clym le devient au propre comme au figuré.

L'aveugle et le séducteur jouent le rôle de bourreau. Toutefois le séducteur est à la fois victime et bourreau car il fascine autant qu'il est fasciné tandis que l'aveugle résiste à la fulguration. Avant d'aborder le groupe des voyeurs, on pourrait d'abord voir ce qui sépare le bourreau-séducteur du bourreau-aveugle.

En premier lieu, on doit remarquer que l'aveugle est le plus souvent cultivé : Henry Knight est un critique littéraire et un essayiste, Angel Clare a fait des études, Swithin est astronome, Clym est plus éduqué que la plupart des habitants d'Egdon. Fitzpiers est lui aussi aveugle par certains côtés. Si l'on examine attentivement les circonstances de la première rencontre de Grace Melbury avec le jeune médecin, on se rend compte que ce dernier ressemble par certains traits à la catégorie des idéalistes au sens péjoratif du terme. Lorsque Grace le voit pour la première fois, Fitzpiers dort sur un canapé :

As soon as the door was shut upon her she looked round the room, and started at perceiving a handsome man snugly ensconced on the couch, like a recumbent figure within some canopied mural tomb of the fifteenth century, except that his hands were not exactly clasped in prayer. [...] Approaching the chimney her back was to Fitzpiers but she could see him in the glass. An indescribable thrill passed through her as she perceived that the eyes of the reflected image were open, gazing wonderingly at her. Under the curious unexpectedness of the sight she became as if spell-bound, almost powerless to turn her head and regard the original. However, by an effort she did turn, when there he lay asleep as before." (Hardy 1998d, 127-128) 
Fitzpiers aperçoit Grace à diverses reprises; ils sont mus l'un vers l'autre avant même d'avoir échangé une parole et il suffit d'attendre la rencontre, le premier face-à-face. Lorsque le hasard les met en présence, ils s'observent à la dérobée. Une personne qui dort est vulnérable en ce sens qu'elle s'offre à la possession visuelle. Le spectateur jouit d'un sentiment momentané de toute-puissance car l'autre ne peut lui renvoyer son propre regard. Ici, le sommeil sépare de façon très symbolique les consciences rivales des deux personnages qui cherchent à se dissimuler plutôt qu'à se livrer.

Chacun vient donc de surprendre obliquement le regard de l'autre par l'intermédiaire du miroir. Cette approche indirecte est le prélude du malentendu qui ne fera que croître entre eux. Grace sort de la pièce pour décider presque aussitôt de revenir sur ses pas. Fitzpiers, entre-temps, s'est réveillé. Il la voit donc venir vers lui alors qu'il eût été dans l'ordre logique des choses de la voir sortir puisqu'en ouvrant les yeux, dans son demisommeil, il a déjà eu l'occasion de la voir dans la pièce : "That he saw her coming instead of going made him ask himself if his first impression of her were not a dream indeed." (Hardy 1998d, 129) Cette confusion dans l'esprit de Fitzpiers est symbolique : rêve ou réalité ? Reflet ou personne réelle ? Il s'agit là d'un trait caractéristique du jeune médecin, de son idéalisme platonicien :

"I fancied in my vision that you stood there", he said, pointing to where she had paused. "I did not see you directly, but reflected in the glass. I thought, what a lovely creature! The design is for once carried out. Nature has at last recovered her lost union with the Idea! My thoughts ran in that direction because I had been reading the work of a transcendental philosopher last night; and I dare say it was the dose of Idealism that I received from it that made me scarcely able to distinguish between reality and fancy" (Hardy 1998d, 132)

Cet idéalisme colore toute la vie affective de Fitzpiers ainsi que sa conception de l'amour. Pour lui, l'objet d'une passion est son prétexte plutôt que sa source et il s'avoue prêt à aimer n'importe quelle femme car, en projetant sur elle tous ses fantasmes, tous ses désirs, i1 ne pourra manquer de l'idéaliser. C'est ce qui se produit dans le processus de la cristallisation: une branche banale, jetée dans les salines de Salzbourg est retirée toute couverte de cristaux, étincelante comme un bijou. Il ne s'éprend donc pas de Grace mais de l'idée qu'il se fait de la jeune fille. Le lecteur sent que leur relation essentielle est faussée dès le départ puisqu'ils communiquent pour la première fois de façon oblique.

Le thème du miroir est souvent lié chez Hardy au thème du double, de l'échec et de la frustration comme si le mal avait partie liée avec la réflexivité et il concerne souvent des intellectuels comme Henry Knight ou Angel Clare. Henry Knight est un critique littéraire bien connu des milieux lettrés londoniens. Il dissèque la nature humaine tout comme Fitzpiers dissèque des cadavres: Henry Knight n'attire Elfride que par sa froideur et son indifférence. Elle veut sentir peser sur elle son regard admiratif et déploie tous ses talents afin de l'éblouir. Elle risque même de se rompre le cou en montant sur un parapet glissant dans le seul but d'attirer son attention. Knight commente ainsi l'incident dans son carnet de notes: "Town-bred girl will utter some moral paradox on fast men, or love. Country Miss adopts the more material media of taking a ghastly fence, whistling, or making your blood run cold by appearing to risk her neck. (Mem. On Endelstow Tower) " (Hardy 1998c, 176). Chaque tentative d'Elfride pour séduire Knight se solde par un échec cuisant. On a même l'impression qu'elle s'acharne d'autant plus qu'elle le sait insensible. Elle oublie Stephen Smith qu'elle avait promis d'épouser à son retour des Indes. Pour séduire le jeune architecte, il lui avait suffi de paraître et son éclat avait sans la moindre difficulté 
subjugué le jeune homme dès le premier regard qu'il avait porté sur elle. Ce triomphe lui semble bien piètre lorsque surgit celui qui refuse de se laisser éblouir :

Stephen fell in love with Elfride by looking at her: Knight by ceasing to do so. [...]

Knight's experience was a complete disproof of the assumption that love always comes by glances of the eye [...]. Not till they were parted, and she had become sublimated in his memory, could he be said to have even attentively regarded her. Thus, having passively gathered up images of her which his mind did not act upon till the cause of them was no longer before him, he appeared to himself to have fallen in love with her soul, which had temporarily assumed its disembodiment to accompany him on his way. (Hardy 1998c, 188-189; ;'est nous qui soulignons)

L'aveugle échappe à l'éblouissement passif puisqu'il ne subit pas l'attrait de la coquetterie mais cette faculté n'est qu'une forme d'orgueil qui le mène à sa perte. Dans son refus d'être asservi, dans son idéal de possession entière et libre de lui-même, il crée de toutes pièces son propre éblouissement en forgeant cette source de lumière qu'il baptise "âme". Cette mauvaise foi provoque invariablement la tragédie car l'aveugle méconnaît toujours l'autre.

Angel Clare commet la même erreur que Knight. Sa première rencontre avec Tess est, à ce titre, très significative. Le hasard les met en présence le jour de la danse du premier mai, à Marlott. Au moment du départ, le regard d'Angel se pose sur Tess Durbeyfield dont les grands yeux semblent lui reprocher faiblement de ne pas l'avoir choisie comme cavalière. En rejoignant ses frères, Angel ne peut s'empêcher de songer à cette jeune inconnue, regrettant de ne pas l'avoir invitée, de ne pas lui avoir demandé son nom. La note est, pour ainsi dire, donnée : Tess et Angel ne se rencontrent que pour se séparer puisque Angel n'a pas su distinguer Tess parmi les autres paysannes: "Pedigree, ancestral skeletons, monumental record, the d'Urberville lineaments, did not help Tess in her life's battle as yet, even to the extent of attracting to her a dancing-partner over the heads of the commonest peasantry. So much for Norman blood unaided by Victorian lucre." (Hardy 2003c, 17) Plus tard, il la condamnera sans appel lorsque les apparences seront contre elle car il sera incapable de discerner la "pureté" morale de Tess.

Clym Yeobright, autre héros de lumière, est lui aussi incapable de comprendre Eustacia : "Ceux qui sont le plus capables de pitié fraternelle pour leur espèce", écrit Jean-Jacques Mayoux, "sont peut-être le moins capables de sentiments aisés, complets et chaleureux pour leurs proches. Clym était fait d'avance pour la prédiction laïque et le célibat. Son histoire ne fait guère que le confirmer dans ce qu'il croit de la condition humaine et des illusions du bonheur. C'est une éducation, payée du sang des autres, de philosophe héraclitien" (Hardy 1947, xiv). Clym recherche inconsciemment une femme qu'il associe tout naturellement à ses projets d'action sociale. Eustacia attend de l'amour une justification de son existence triste et solitaire; d'un amant, qu'il l'emmène loin de cette région d'Egdon qu'elle hait du plus profond de son être. Il n'en reste pas moins que tout commence au moment où Clym voit Eustacia sous son masque et que, si cette dernière ne s'était pas volontairement faite objet fascinant, rien ne serait arrivé. C'est au coucher du soleil que le lecteur voit d'abord Eustacia épier les abords de la maison des Yeobright lorsque la nouvelle du retour de Clym lui parvient. C'est pour elle l'occasion tant attendue de s'échapper de cette Lande où elle se sent exilée. Clym peut revenir à Paris; tout est possible avec lui. Elle est tout d'abord charmée par la voix du jeune homme le soir de son retour car elle ne peut distinguer son visage de l'endroit où elle se tient cachée. Ses tentatives pour le rencontrer échouent. Le soir de Noël, elle décide de se rendre à la fête que donne Mrs Yeobright en l'honneur de son fils. Comme elle n'est pas invitée, elle se 
mêle à la troupe des masques sous le déguisement du Chevalier Turc. Son rôle terminé, gisant parmi les occis, son regard cherche à travers les rubans de sa visière, dans la lueur des chandelles, à distinguer Clym Yeobright. Lorsque les autres masques enlèvent leur casque afin de boire et de manger, Eustacia est obligée de garder le sien de peur d'être découverte. Clym est intrigué, puis fasciné :

When the disguised Queen of Love appeared before Aeneas a preternatural perfume accompanied her presence and betrayed her quality. If such a mysterious emanation ever was projected by the emotions of an earthly woman upon their object, it must have signified Eustacia's presence to Yeobright now. He looked at her wistfully, then seemed to fall into a reverie, as if he were forgetting what he observed. (Hardy 1999, 141-142)

Eustacia tombe amoureuse d'une voix sans visage, Clym d'une créature masquée. Aucun ne connaît le vrai visage de l'autre, d'où le malentendu qui ne fera que croître. Eustacia tend de tout son être à s'éloigner de la Lande tandis que Clym a décidé de revenir pour toujours à cette terre ingrate. Il idéalise Eustacia, s'obstinant à voir en elle la compagne dont il a besoin pour mener à bien ses projets. Il refuse de voir à quel point la jeune femme hait ce pays austère. Il ne tient pas compte de ses désirs, de sa nature impulsive et sensuelle. Il déclare naïvement à sa mère : "She is excellently educated and would make a good matron in a boarding-school." (Hardy 1999, 191) Mrs Yeobright essaye en vain de lui ouvrir les yeux: "You are blinded, Clym [...] it was a bad day for you when you first set eyes on her. And your scheme is merely a castle in the air built on purpose to justify this folly which has seized you" (Hardy 1999, 191).

Clym, Knight, Angel commettent la même erreur : ils s'éprennent d'une idée forgée de toutes pièces par leur cerveau et ne respectent aucunement la femme qui, de ce fait, devient leur victime. Leur inhumanité prend l'aspect d'une cécité. Si les séducteurs tels que Troy ou Alec sont autant bourreaux que victimes car ils éblouissent tout en étant euxmêmes éblouis, en revanche, les aveugles tels que Clym ou Angel font bien plus souffrir qu'ils ne souffrent. Le narrateur déclare, par exemple, que l'amour de Clare pour Tess "was ethereal to a fault, imaginative to impracticability. With these natures, corporeal presence is something less appealing than corporeal absence; the latter creating an ideal presence that conveniently drops the defects of the real." (Hardy 2003c, 244)

Ce type de personnage s'oppose à la fois au séducteur, c'est-à-dire au type du "woman's man" et au groupe de héros que l'on a qualifiés de voyeurs et auquel appartiennent Gabriel Oak, George Somerset, Diggory Venn, Edward Springrove, Stephen Smith, Christopher Julian et bien d'autres. Ces derniers se tiennent le plus souvent à l'écart, en spectateurs. Si l'on compare la première rencontre de Bathsheba Everdene avec Gabriel aux deux premières scènes où la jeune fille se trouve confrontée avec Troy par exemple, ou bien à la façon dont elle fait irruption dans la vie de William Boldwood, on comprend du même coup la différence qui sépare Gabriel de ses deux rivaux.

Le roman commence par la découverte fortuite de Bathsheba par Oak. Ce dernier épie la jeune fille sans que celle-ci s'en aperçoive. Elle est en train de contempler son visage dans un miroir, se souriant à elle-même :

What possessed her to indulge in such a performance in the sight of the sparrows, blackbirds, and unperceived farmer, who were alone its spectators - whether the smile began as a factitious one to test her capacity in that art, - nobody knows: it ended certainly in a real smile; she blushed at herself, and seeing her reflection blush, blushed the more.

[...] Woman's prescriptive infirmity had stalked into the sunlight, which had 
invested it with the freshness of an originality. [...] She simply observed herself as a fair product of Nature in a feminine direction - her expression seeming to glide into far-off though likely dramas in which men would play a part - vistas of probable triumphs - the smiles being of a phase suggesting that hearts were imagined as lost and won. (Hardy 2003a, 6)

J. Hillis Miller commente ainsi ce passage :

Bathsheba's charming vanity, smiling at herself, blushing at the smile, and blushing more at her perception of the blush, in echoing self-reflection, brings into the open the hidden intimacy of her relation to herself. The act is more provocative even than seeing her unclothed would be, for it reveals a spiritual rather than a physical mystery. [...] Gabriel, meanwhile, like the narrator and the reader, has been watching her all along, as David in the biblical story watched Bathsheba bathing (2 Samuel II:2). Gabriel has been stealing from her that sovereignty over herself she has thought she had been enjoying in secrecy and security. It is not surprising that Gabriel is entranced by his unintentional entry into this arcanum of the feminine nature. Enjoying the delicate peeping-Tom pleasure of seeing without being seen is a pleasure tantalizingly attractive to Hardy's people. Gabriel is captivated in an instant, and the permanent structure of his relation to Bathsheba is established. For most of the novel he watches over her from a distance as her servant and unrequited lover. (Hillis Miller 123-124)

On peut ajouter au commentaire de J. Hillis Miller quelques remarques portant sur la nature des rapports visuels entre les personnages concernés. La supériorité de Gabriel Oak sur Boldwood et Troy vient de sa situation physique privilégiée au départ. Boldwood laisse l'ennemi pénétrer dans sa forteresse tandis que Gabriel l'observe à la dérobée, derrière une haie. Il n'y a pas d'échange de regards : il n'est pas fasciné comme Troy. En effet, lorsque la jeune coquette surgit dans son champ visuel, il n'est nullement victime d'une extase destructrice car elle lui dévoile son moi le plus intime sans le vouloir. La chose visible ne fascine que par le mystère qu'elle semble voiler. Dans "fasciner", il y a fascinum qui signifie charme, maléfice. Gabriel conjure le sort dès le départ en restant à l'abri et sa réflexion s'exerce tout à loisir au cours de cette première apparition : "Gabriel [...] glanced back to where he had witnessed her performance over the hedge, and said 'Vanity'." (Hardy 2003a, 7) Il n'y a donc pas d'aveuglement fatal puisqu'en surprenant la façon dont elle s'y prend afin de séduire par son sourire enjôleur, il conjure le pouvoir ensorceleur de la jeune fille. Gabriel restera tout au long du roman à une distance respectueuse de Bathsheba. Il ne devra son salut qu'à cette distance salutaire: Boldwood et Troy se damnent, le premier pour avoir imprudemment répondu à la séductrice, le second pour ne pas avoir su perpétuer l'éblouissement subi en se dispersant en une multiplicité de ravissements fugitifs.

50 L'attitude de Gabriel Oak est comparable à celle de Diggory Venn, l'un des héros de The Return of the Native. Venn est le témoin de chaque phase du drame qui se déroule sur Egdon Heath. C'est avec les yeux de l'homme au rouge que le lecteur découvre, dans la nuit éclairée de flammes, la silhouette d'Eustacia se détachant sur l'obscurité, ombre parmi les ombres. Ce voyeur dissimulé est le bon génie de la lande : il veille à distance sur Thomasin tout comme Gabriel veille sur Bathsheba. La générosité de Diggory est aussi grande que sa discrétion. Il met tout en œuvre afin que celle qu'il aime puisse épouser l'inflammable Wildeve. Ce dernier est victime d'une succession d'éblouissements discontinus. Grâce aux efforts de Venn, Thomasin finit par épouser Wildeve. Il est vrai que Venn devait disparaître, une fois sa mission accomplie. On doit cependant remarquer que ce voyeur bienfaisant et bienveillant est le seul à être épargné à la fin du roman. Dissimulé dans l'ombre complice de la Lande, son regard reste obstinément fixé sur les 
faits et gestes des autres protagonistes du drame et jamais sur lui-même. Venn échappe au sort tragique de Wildeve de même que Gabriel finit par l'emporter sur ses deux rivaux, Springrove sur Aeneas, Somerset sur De Stancy.

Chaque personnage, peut-on dire, a une attitude visuelle bien spécifique qui définit son comportement et son sort ${ }^{6}$. Comme le désir éveillé par le regard ne trouve pas de quoi se satisfaire dans l'acte de voir, les héros se damnent en essayant d'abolir la distance ou de surmonter les obstacles qui les séparent de l'objet aimé. Le seul moyen d'éviter le pire est de maintenir la distance, de l'accepter, de s'y soumettre. La possession par le regard à distance ne connaît pas la mort du désir qui est la loi de toute possession. On doit remarquer que les héros soumis à la loi du regard sont incapables de transformer la fascination instantanée en dévotion durable: ils sont infidèles dès qu'un nouvel éblouissement survient. Bathsheba et Felice sont abandonnées dès que le désir de Troy et de Fitzpiers est satisfait. Troy se détache de Bathsheba aussitôt après leur mariage et s'adonne à la boisson en compagnie des ouvriers. Lorsque Troy revoit Fanny dans son cercueil, sa passion renaît car la mort la lui rend à jamais inaccessible. De même, il reparaît devant Bathsheba le soir où Boldwood doit annoncer leurs fiançailles. Fitzpiers délaisse Mrs Charmond lorsqu'elle lui a donné tout ce qu'il pouvait souhaiter.

Dans la mesure où le voyeur échappe aux tribulations qui sont le lot des victimes de la fascination, on pourrait penser que l'éros visuel est une apologie du voyeurisme tant il est vrai que le voyeur est souvent le vainqueur ou, du moins, celui qui parvient à survivre. La distance le préserve de l'humiliation car on ne peut lui refuser ce contact à distance dont il jouit à la dérobée. L'objet ne se trouve pas toujours dans son champ visuel? Qu'à cela ne tienne: la mémoire recrée la figure désirée et l'image mentale prend le relais de la sensation actuelle. On peut ainsi lire dans A Pair of Blue Eyes :

Every woman who makes a permanent impression on a man is afterwards recalled to his mind's eye as she appeared in one particular scene, which seems ordained to be her special medium of manifestation throughout the pages of his memory. As the patron Saint has her attitude and accessories in mediaeval illumination, so the Sweetheart may be said to have hers upon the table of her true Love's fancy, without which she is rarely introduced there except by effort; and this though she may, on further acquaintance, have been observed in many other phases which one would imagine to be far more appropriate to love's young dream.

Miss Elfride's image chose the form in which she was beheld during these minutes of singing for her permanent attitude of visitation to Stephen's eyes during his sleeping and waking hours in after days. (Hardy 1998c, 22).

Stephen n'est pas le seul héros qui ait une prédilection pour ce genre de fantasme où l'image de la bien-aimée est recréée avec une foule de détails. Ces personnages sont tantôt voyeurs, tantôt spectateurs de leur propre vision composée à l'aide de la mémoire chaque fois qu'ils le désirent. Le plaisir que procure une telle rêverie vient de ce qu'elle rend l'objet convoité bien plus docile et accessible puisqu'on fait naître en soi le spectacle désiré chaque fois qu'on en éprouve le besoin. Roland Barthes souligne le même phénomène à propos de 1'“Eros racinien" :

L'imagination est toujours rétrospective et le souvenir a toujours l'acuité d'une image, voilà le protocole qui règle l'échange du réel et de l'irréel. La naissance de l'amour est rappelée comme une véritable "scène" : le souvenir est si bien ordonné qu'il est parfaitement disponible, on peut le rappeler à loisir, avec la plus grande 
chance d'efficacité. [...] Il y a là comme une sorte de transe : le passé redevient présent sans cesser pourtant d'être organisé comme un souvenir: le sujet vit la scène sans être submergé ni déçu par elle. La rhétorique classique possédait une figure pour exprimer cette imagination du passé, c'était l'hypotypose [...] ; l'image tient lieu de la chose: on ne peut mieux définir le fantasme. Ces scènes érotiques sont en effet de véritables fantasmes, rappelés pour alimenter le plaisir ou l'aigreur, et soumis à tout un protocole de répétition (Barthes 22-23, c'est l'auteur qui souligne). puisque l'histoire commence avec lui. La différence de sort entre les voyeurs... et les autres est importante. Diggory Venn ou Gabriel Oak sont caractérisés par leur don d'ubiquité : ils surgissent toujours au "bon moment", lorsqu'on a besoin d'eux, et se tiennent à l'écart le reste du temps. Ce voyeurisme doublé d'ubiquité en fait des dieux invisibles tandis que les victimes de la fascination ressemblent étrangement aux humbles mortels. Si l'on passe en revue la production romanesque, on s'aperçoit que le voyeur se fait de plus en plus rare puisque le dernier "voyeur heureux" se trouve dans A Laodicean (1881). Il s'agit de George Somerset qui découvre Paula au "bain", telle Bethsabée ("objet de désir") surprise par David. Oak et Somerset se ressemblent : Oak surprend Bathsheba au moment où elle s'admire dans un miroir. Dans les deux cas, le voyeur ne voit qu'un reflet. Toutefois, contrairement à Actéon transformé en cerf et déchiqueté "par ses propres chiens" pour avoir surpris Diane au bain, Oak n'est pas aliéné au point de devenir la proie de ses instincts. Il ne se montre pas, évitant par là même de provoquer le courroux de la belle. Lorsque, un moment après, i1 acquitte pour elle le droit de péage, elle le toise avec mépris. Cet argent est en fait le prix du spectacle: Oak n'est pas un resquilleur. Jude ne sera pas aussi fortuné puisqu'il se laissera écarteler par l'autre (Arabella) et par son même, Sue (avatar de l'épisode biblique bien connu "Susannah and the Elders") qui laisse dans son sillage un nombre impressionnant de victimes.

Le triomphe du voyeur signifierait la fin du règne de l'illusion. Toutefois, cet avènement du regard "divin" semble compromis. Venn ne devait pas épouser Thomasin : cette fin a remplacé un premier dénouement où Venn disparaissait sur la lande.

Lènement du temps humain coïncide donc avec le regard désirant. Si ce premier regard a souvent pour "cadre" une fenêtre, c'est parce qu'il s'agit du lieu tragique par excellence. En effet, la fenêtre est symbole d'ouvert et de fermé, de dedans et de dehors à la fois. En elle se trouve donc abolie toute notion d'extériorité, d'ailleurs. Rien d'étonnant à ce qu'elle figure sans cesse dans les rapports visuels. Comme pour Adam, tout 
commence pour les héros à l'instant où leurs yeux se posent sur une "fem-être". Ceux qui survivent sont précisément ceux qui résistent à la tentation. Ceux à qui le regard ne suffit pas passent à l'acte et périssent ; l'acte, c'est-à-dire, l'enfant, le germe de la mortalité Little Father Time.

\section{La fem-être}

57 Certaines héroines hardyennes passent de longues heures derrière leur fenêtre. Elles scrutent l'horizon, attendant l'événement. La monotonie de leur existence quotidienne serait difficilement supportable si elles ne disposaient de ce poste d'observation idéal car, comme le dit si bien Jean Rousset à propos des personnages flaubertiens : "La fenêtre unit la fermeture et l'ouverture, l'entrave et l'envol, la clôture dans la chambre et l'expansion au dehors" (Rousset 123). Anne, Elizabeth-Jane, Fancy scrutent inlassablement l'horizon du haut de leur "vigie". Lucetta loue une maison dont les fenêtres donnent sur la place de Casterbridge afin de tromper son ennui. Le grand-père d'Eustacia et le marin Newson choisissent une maison d'où ils peuvent apercevoir "la mer". Le père d'Elisabeth-Jane préfère quitter Casterbridge où pourtant réside le seul être qui lui soit cher pour aller s'installer à Budmouth.

La fenêtre est donc le moyen de garder un contact visuel avec un objet hors d'atteinte. Cependant, elle devient vite symbole de frustration: l'objet aimé inaccessible est visible de l'extérieur lorsque les rideaux ne sont pas tirés et l'on assiste alors à des scènes douloureuses. Les regards de l'espion caché dans l'obscurité restent fixés sur ce point lumineux qui le fascine : pensons à Dick, à Jude et à bien d'autres regards qui convoitent et souffrent en silence de l'autre côté de la fenêtre. Pensons au douloureux regard de Mrs Yeobright qui sent son fils lui échapper (Hardy 1999, 279), à l'amertume de Jude devant les fenêtres des colleges où il ne pourra jamais entrer (Hardy 1998b, 328). La fenêtre ne se contente donc pas de frustrer puisqu'elle permet aussi de guetter, d'espionner sans être vu. Chez Hardy, rien ne reste secret de ce que l'on aurait voulu cacher. En fait, ce thème du "secret" rejoint celui du connu/inconnu, caché/visible que nous trouvons dans toute l'œuvre. Si les murs ont des yeux et des oreilles, c'est que l'ennemi est dedans et dehors, en l'homme et à l'extérieur de chaque être. Il n'y a pas de héros victime de forces purement extérieures. Les erreurs commises par l'assassin Manston dans Desperate Remedies rendent visible ce qu'il voulait garder "caché". En fait, tout ce premier roman de Hardy a pour thème l'absence de frontière entre extériorité et intériorité puisque le meurtre et le suicide ne sont qu'un seul et même acte. Manston croit éliminer la source de son "mal" en tuant sa femme (Eunice : l'eunuque) alors que ce mal est constitutif de son être et c'est lui-même qu'il tue. Voilà pourquoi l'œuvre fourmille d'yeux qui sont à l'affût du moindre "faux-pas" de l'autre.

La fenêtre est surtout un poste d'observation idéal, vigie sur la rue d'où va surgir sa prochaine victime. Relisons plutôt le passage qui suit le départ du pauvre Dick sous la pluie :

'I like Dick, and I love him; but how poor and mean a man looks in the rain, with no umbrella, and wet through!'

As he vanished, she made as if to descend from her seat; but glancing in the other direction she saw another form coming along the same path. It was also that of a man. $\mathrm{He}$, too, was in black from top to toe; but he carried an umbrella.

[...] He passed in due time directly beneath her, and in looking down upon the 
exterior of his umbrella her feminine eyes instinctively perceived it to be of superior silk, and of elegant make. (Hardy 2004, 136)

Il est impossible de dénombrer les allusions aux fenêtres dans tous les romans. Il y en a, par exemple, environ soixante-dix dans The Mayor of Casterbridge où chaque personnage, pour ainsi dire, a sa fenêtre : Elizabeth-Jane change même de chambre au cours du roman afin de changer de poste d'observation. Lorsqu'un personnage est sans domicile (ce qui est le cas de Farfrae, d'Elizabeth et de sa mère au début du roman), les fenêtres des édifices publics prennent la relève : c'est par la fenêtre de l'Hôtel des Armes Royales que Susan revoit son mari pour la première fois et que Farfrae communique avec Henchard. Farfrae descend à l'auberge des Trois Matelots, Susan et sa fille aussi. Henchard vient voir Farfrae : les deux femmes écoutent leur conversation derrière la cloison. Henchard ne réussit pas à convaincre Farfrae de rester. Il prend congé de l'Écossais mais revient près de l'auberge. Le thème de la fenêtre se mêle parfois aussi au pouvoir maléfique de la musique, quand on sait le rôle fatal que jouera Farfrae dans la vie de Henchard, on réalise qu'il aurait mieux valu pour le Maire de pas être vu par Donald dont la voix le charme tant. Il sort de sa réserve et s'entiche de cet étranger qui le dépossédera de tous ses biens les plus chers.

Le rôle que joue la fenêtre de l'Hôtel des Armes Royales est particulièrement remarquable. Cette fenêtre cintrée sépare les protagonistes: Henchard et les notables de la ville sont vus par un groupe de petites gens. L'éloignement produit une distorsion optique suivant la place occupée par le spectateur. Cette perspective déformante provenant de la distance qui sépare ici le spectacle des spectateurs souligne la nature des rapports qui lient les personnages. La vision qui s'offre à Susan Henchard est la nouvelle image que son mari tient à imposer au monde : celle d'un homme digne, respectable dans l'exercice de ses fonctions de Maire de la ville. Le lecteur a l'impression d'assister à une représentation figée, artificielle, les acteurs étant de l'autre côté de la fenêtre qui sert de cadre ou de scène, et les badauds faisant office de public. Une scène semblable se déroule bien plus tard après la déchéance de Henchard lorsque Elizabeth-Jane apprend par hasard la ruine de son beau-père en passant devant l'Hôtel des Armes Royales. La même disposition, les mêmes effets de distance sont repris mais les positions sont inversées : ce n'est plus Elizabeth-Jane qui contemple de l'extérieur un riche parent semblant se mouvoir dans une sphère supérieure à la sienne mais Henchard qui comprend l'erreur de son comportement envers la jeune fille: il se rend compte qu'elle était son seul bien véritable, sa seule richesse et qu'il est responsable de la perte de cette affection. L'inflammable Lucetta se regarde malgré elle passer dans la rue sous la forme d'une effigie et meurt peu après d'une fausse couche, dédoublement mortifère qui tuera aussi Henchard. Eux qui voulaient s'élever dans l'échelle sociale afin d'être le point de mire de Casterbridge meurent en quelque sorte d'un "excès de regards" dramatisé lors de la skimmity-ride. À l'élévation succède la chute d'Icare, autre victime célèbre de la mégalomanie, comme si nul ne pouvait échapper à la compulsion de répétition qui génère le récit. En vendant sa femme et son enfant, Henchard commet un forfait qu'il ne cessera de payer. Voilà pourquoi le lieu de foire se nomme "Weydon Priors" : il indique au lecteur que les dés sont jetés, "the way is done before", pour le héros tragique. Le même sème de fermeture se retrouve dans l'amphithéâtre romain de Casterbridge, "The Ring", qui connote l'idée d'alliance pour le moins parodique : comment réparer l'irréparable, qu'il s'agisse de blé corrompu ou de femme vendue? 
Certains personnages passent de longues heures dans l'obscurité, les yeux fixés sur la fenêtre éclairée de l'objet aimé. Cette fascination s'explique dans la mesure où cela leur permet de suppléer à un échange de regards, à un face-à-face ou bien un lieu de fuite lorsqu'on veut se soustraire au regard de l'autre. Si ces échanges de regards sans contact des corps, ces approches furtives aussitôt éludées ont pour cadre une fenêtre, c'est parce qu'elle est le lieu d'une ouverture sur le monde extérieur, d'intimité à distance, mais aussi lieu de fuite, symbole de frustration, d'obstacle à toute communication véritable, d'éloignement, de vision déformée, d'incertitude sur le réel, d'effets de mirage et d'illusions. C'est le point de départ des relations qui s'établissent entre les protagonistes : dialogue sans paroles qui se réduit à un échange de regards lourds de conséquences. Ouverte ou fermée, elle est à proprement parler néfaste car c'est par elle que l'être s'échappe, se dissout, se fond, se perd, piège qui est en fait l'emblème du trou au fond duquel gisent la femme, la nuit et la mort ${ }^{7}$.

\section{Microcosme et macrocosme}

Cette analyse du regard serait incomplète si nous n'élargissions notre propre champ de vision puisque, à chaque page, nous constatons que microcosme et macrocosme sont indissociables. La lumière de l'œil lie, pour ainsi dire, la créature au cosmos dans la mesure où le regard est isomorphe du rayon lumineux. Mais lisons plutôt cette description du soleil dans Tess of the d'Urbervilles:

The sun, on account of the mist, had a curious sentient, personal look, demanding the masculine pronoun for its adequate expression. His present aspect, coupled with the lack of all human forms in the scene, explained the old-time heliolatries in a moment. One could feel that a saner religion had never prevailed under the sky. The luminary was a golden-haired, beaming-faced, mild-eyed, God-like creature, gazing down in the vigour and intentness of youth upon an earth that was brimming with interest for him."(Hardy 2003c, 86)

Le souverain regard solaire ${ }^{8}$ doté, comme il se doit, des attributs traditionnels du mâle, courtise ici une terre pleine comme une coupe sur le point de déborder. Nous avons là un saisissant raccourci des liens tissés entre le haut et le bas, entre ciel et terre car la focalisation solaire n'est pas que lumière. Elle est aussi chaleur. Eustacia joue avec le feu sur la lande et périt, victime à la fois du feu et de l'eau; du feu, sous la forme d'une figurine de cire percée d'épingles, et de l'eau puisqu'elle se noie dans l'écluse. Cette cosmisation du regard opère une dichotomie entre lumière et feu, entre feu dionysiaque et feu apollinien sur laquelle vient se greffer le couple terre/eau qui joue lui aussi un rôle important dans la dialectique vie/mort. Prenons, par exemple, le passage où Gabriel Oak s'interroge sur son avenir après la perte irréparable de son troupeau, catastrophe (rappelons-le) provoquée par l'impétuosité de son chien :

Oak raised his head, and wondering what he could do, listlessly surveyed the scene. By the outer margin of the pit was an oval pond, and over it hung the attenuated skeleton of a chrome-yellow moon, which had only a few days to last - the morning star dogging her on the right hand. The pool glittered like a dead man's eye, and as the world awoke a breeze blew, shaking and elongating the reflection of the moon without breaking it, and turning the image of the star to a phosphoric streak upon the water. (Hardy 2003a, 33 ; c'est nous qui soulignons). la partie en allant jouer de la flûte dans les rues de Casterbridge avant de se faire 
embaucher comme berger. L'argent gagné avec la flûte lui permet de s'offrir un repas : en d'autres termes, il fait face à la tentation de la dissolution, du retour au néant, du vide par le "plein", en se nourrissant. Ce geste symbolique s'inscrit dans le droit fil de son "voyeurisme" qui est avant tout une mesure de conservation dans un univers où font rage les forces de dissolution.

Si l'œil est lumière, le soleil est un œil souvent impitoyable ainsi que l'atteste, par exemple, l'épisode de la Falaise dans A Pair of Blue Eyes (Hardy 1998c, 211-220). Rien ne manque au tableau: Henry Knight qui a perdu son "chapeau" se retrouve accroché à la paroi d'une falaise, prisonnier entre mer et ciel. Verticalité précaire car, à la moindre défaillance, il sera absorbé par l'élément liquide horizontal qui git sous lui. Telle est la fragile condition humaine! Cette dialectique de l'horizontal et du vertical se joue sur l'antagonisme ténèbres/lumière où se retrouve le regard par le truchement de l'aveuglement ou de la fulguration qui plongent ceux qui en sont victimes dans la nuit absolue figurée tantôt par l'élément liquide, tantôt par la terre.

Dans notre chapitre final, nous étudierons les implications idéologiques de cette configuration basée sur les deux dynamismes antithétiques et inséparables que constituent l'irradiation et l'absorption par le truchement des relations isomorphiques établies entre regard et lumière, soleil et œil, regard et feu, soleil et feu, œil et ciel, ciel et liquide, eau et nuit, terre et ténèbres. Comme chez la plupart des romantiques, l'alliance nuit/tempête (du chaos intérieur) se combine à une image de dynamisme hydrique où le liquide vient figurer l'écoulement et la fusion, symboles de la perte d'identité. Mais comment ne pas y voir aussi les préoccupations d'une époque soucieuse d'énergie et de circulation de l'énergie ainsi que le rappelle Michel Serres dans l'ouvrage qu'il a consacré à Zola (Serres 1975). Feu et eau jouent ici un rôle primordial dans un mouvement d'échange perpétuel entre ciel et terre car, ne l'oublions pas, la vapeur d'eau de la locomotive est aussi celle des nuages électriques qui restituent l'eau à une terre asséchée par le soleil.

On voit se profiler une théorie élémentaire des relations à travers cette cosmisation des rapports humains où l'on discerne les valeurs symboliques du feu et de la lumière selon que le regard est lié à l'un ou à l'autre domaine du psychisme. Le regard du voyeur est une pure luminescence, symbole du contrôle de l'intellect, par opposition à la fulgurance, symbole d'un embrasement affectif non contrôlé. Un trajet se dessine qui relie en quelque sorte le soleil à la mer ainsi que nous pouvons le voir à travers cette description de l'héroïne de A Pair of Blue Eyes, roman dont l'action se situe au bord de la mer, dans un petit village au nom évocateur de fin du monde : "Endelstow". Elfride meurt victime de l'indifférence de Knight, héros solaire par son idéalisme platonicien, et de la vindicte de Mrs Jethway. Elfride est un nuage pris entre soleil et mer. Cette double nature aérienne et hydrique est conforme à un être aérien : n'est-elle pas un "elfe" qui, comme chacun sait, a l'étonnant pouvoir de s'évanouir dans les airs? Elfride va se laisser dissoudre par le regard platonicien de Knight qui, assez curieusement, découvre en Mrs Jethway une alliée, comme si le soleil et la mer se liguaient pour venir à bout d'un nuage, emblème de la personnalité fragile et incertaine de la jeune femme. La mort d'Elfride devient l'emblème de la tragédie humaine: l'homme, être de mouvance qui rêve de lumière depuis sa prison de chair opaque. Telle est l'image véhiculée à la fin par la description du cercueil d'Elfride sur lequel on peut lire :

ELFRIDE

WIFE OF SPENSER HUGO LUXELLIAN, 
FIFTEENTH BARON LUXELLIAN :

DIED FEBRUARY 10, 1867. (Hardy 1998c, 375)

69 solaires effracteurs. Ses yeux bleus, symboles d'une invite à un devenir symbiotique, se fossilisent sous l'action minéralisante des acteurs mâles auxquels elle est confrontée : son goutteux de père, la tombe de Felix, l'église que répare Stephen l'architecte, la plumeépée du chevalier-gisant Knight et la lumière phallique de Lord Luxellian, lux-alien, le lumineux, l'étranger, le veuf, le consolable, le prince du Wessex à la tour achevée. Retour au Nom-du-Père, à la Loi, à la pierre, à l'un, des atomes, du nuage, du flux, du vivant, de la multiplicité. Pierre et lumière se liguent également contre Tess, symbole du flux, du liquide, du mouvement, de la marche, du vivant et dont la course s'achève dans la tombe près des gisants d'Urberville. Cette minéralisation au Nom-du-Père, de l'ancêtre de la lignée patriarcale est aussi une immolation sur la pierre-autel du sacrifice à Stonehenge, temple païen où l'on célébrait jadis le culte du soleil. Cette dessiccation n'épargne pas davantage Jude dit "Jude l'Obscur" qui meurt lui aussi d'une "réduction de volume", d'une mise à plat mortifère. Il passe et repasse devant la borne portant ses initiales et son index pointé droit devant, aiguille de pierre à jamais figée en direction du Père, de Phillotson, le collectionneur de ruines romaines, Phillotson ou celui qui aime le fils, ou la pierre (son/ stone). Phillotson qui, en partant pour Christminster, offre à Jude, au fils, le livre du Dauphin, don pétrifiant du maître d'école de Lumsdon (alias le maître des taudis !) : "the old, superseded, Delphin editions [...] roughened with stone-dust", livre pétrifié que le lecteur retrouve près du cadavre raidi, "straight as an arrow". (Hardy 1998b, 407)

Chaque roman ou presque offre au lecteur une spectralisation opérée par une lapidation ou une fossilisation suivie d'une petite visite au tombeau ${ }^{9}$, comme si le roman suivant ne pouvait s'écrire que sur le blanc de la pierre tombale du roman précédent. Blanc de la pierre, de la page, de l'ange annonciateur, de Gabriel Oak qui, lui, a le rare privilège d'épouser Bathsheba tandis que Troy a réduit Fanny a un nom propre sur une pierre tombale, emblème de la réduction mortifère de l'un. En restant à l'écart, Oak n'a pas cherché la fusion. Il symbolise donc le chêne de la survie, l'arbre où la sève va continuer de couler. Il n'en va pas de même avec Boldwood que sa hardiesse précipite dans le cachot, bold/wood ou le bois qui meurt d'ankylose, prisonnier de la pierre carcérale.

71 Le respect de la distance/différence ${ }^{10}$ prend des formes diverses dans le texte romanesque : altruisme et fidélité sont en général l'apanage de ceux qui en font preuve par opposition aux égocentristes que leurs passions précipitent au fond de l'abîme. Ces derniers perdent leur identité en raison de leur démesure qui est, précisément, une méconnaissance de la distance, de la mesure, de l'écart irréductible. Il s'agit là à proprement parler du scénario mis en scène par les rapports visuels constitutifs du "Chemin du Désir" qui s'ouvre sur la fem-être et s'achève dans la tombe. La cosmisation des rapports humains s'intègre à ce dispositif sémiotique du leurre en ce sens qu'il n'y a pas de rupture entre microcosme et macrocosme mais un seul et même événement tragique auquel sont confrontés les sages voyeurs, les méchants fascinants et les victimes fascinées. La dessiccation et la pétrification sont les deux formes que prennent dans le texte les forces meurtrières qui sont liguées contre le flux, le flot, le liquide, le vivant. 
72 Il n'y a pas de cause première ni d'enchaînement fatal mais un simple jeu de hasard suivi, ensuite, d'effets logiques. L'existence du voyeur, aux côtés du séducteur, pose une alternative, un choix. L'erreur de départ est une erreur de jugement rendue métaphoriquement par l'erreur visuelle. Le héros croit saisir l'objet de son désir et se retrouve les mains vides. La quête d'unité, de fusion est illusoire et néfaste. Egbert Mayne croit s'unir à Geraldine ; Aeneas croit s'unir à Cytherea ; Dick croit s'unir à Fancy Day, la chimère lumineuse comme le soleil, alors qu'il y aura toujours entre eux ce secret auquel il n'aura pas accès. Felix, Stephen, Knight et Lord Luxellian ne peuvent pas davantage s'unir à Elfride. Boldwood, Troy, Giles, Wildeve, Jude et bien d'autres meurent aussi victimes de leur désir.

The Well-Beloved, enfin, nous propose deux versions différentes quant au dénouement. Dans la première, Jocelyn Pierston met fin à ses jours en se noyant tandis que, dans la seconde, il finit par épouser Marcia que l'on amène à l'Église sur un fauteuil roulant parce que ses rhumatismes l'empêchent de marcher : "She stood the image and superscription of Age - an old woman, pale and shrivelled, her forehead ploughed, her cheek hollow, her hair white as snow." (Hardy 1997, 332) Marcia évoque ici l'image de la mort : elle est l'autre d'Avice que son union avec Pierston transforme en même de Pierston. Ce mariage avec une femme en ruine est l'alternative de la fusion/noyade. Retour à la mer(e) ou à la poussière de la ruine. Dans les deux cas, c'est la même étreinte avec la mort. L'autre de l'Autre s'est évanoui et il ne reste que le cadavre du Même. Cette femme en ruine n'est qu'un gisant, un signe, une absence que rien ne viendra combler. D'Egbert Mayne à Jocelyn Pierston, en passant par Jude, il s'agit toujours du même désir narcissique de fusion avec une image de double créée par le moi totalitaire. Cette figure de double se superpose à l'autre et le tue : telle est la manifestation ultime de la perversion de l'Un.

Il en va de la femme comme de l'œuvre d'art. La description de Marcia, à la fin de The Well-Beloved, ne laisse planer aucun doute sur cette assimilation : "To this the face he once kissed had been brought by the raspings, chisellings, scourgings, bakings, freezings of forty invidious years" (Hardy 1997, 332). Tant que le sculpteur Pierston se contente de vouloir s'unir à l'une des trois Avice, celle-ci se perpétue de mère en fille. Sa beauté traverse les ans sans subir le moindre outrage. Lorsqu'il décide, enfin, de "se ranger" en épousant Marcia, il se trouve confronté à une femme "en ruine". La pétrification qu'a subie Marcia vient du mal que lui a fait Pierston (le "sculpteur Perce-pierre") en l'abandonnant pour se lancer à la poursuite de sa chimère préférée, Avice Caro. Femme, statue ou poème, il s'agit toujours d'une forme qu'un artiste cherche à capturer avec un instrument phallique, ciseau ou plume. Femme en ruine ou ébauche ratée, telle est celle avec laquelle le moi s'unit, devenu lui-même un vieillard impuissant. La fusion est donc la mort de l'autre devenu "le même" sous le regard phallique prédateur. Belle union en vérité que celle de ces deux gisants qui ne pourront sans doute plus que se regarder ... en chiens de faïence. Cette dernière séquence résume admirablement le triomphe de la pétrification sur l'essor, le mouvement de la quête, de l'errance, du désir.

L'éros visuel est donc, à proprement parler, la forme que prend dans l'œuvre la quête passionnée de l'Un indivisible. L'échange oculaire entre les créatures vient se doubler de regards cosmiques figurant l'échange d'énergie microcosmique et macrocosmique car l'attraction qui régit les rapports des êtres ordonne aussi les rapports des astres ainsi 
qu'en témoignent les nombreuses allusions aux phénomènes atmosphériques, électriques ou magnétiques. L'aspiration verticale à l'un de l'aveugle platonicien s'oppose à l'acceptation du voyeur panthéiste en accord parfait avec le milieu ambiant. Diggory Venn ou Gabriel Oak en sont les plus beaux représentants par leur amour oblatif, symbolisé par leur passivité, leur présence discrète mais enrichissante, l'échange constant qu'ils vivent avec les êtres et les éléments qui les entourent. Le secret de leur bonheur vient de ce qu'ils acceptent le devenir terrestre, le cycle des saisons, la temporalité, la vie et la mort. L'erreur des aveugles vient précisément de leur désir d'absolu qui est un refus du devenir ${ }^{11}$. Lorsque l'extase s'achève, ils retombent lourdement, victimes de leur tunique de Nessus, de leur carcan de chair qui les divise. Prisonniers du temps par la loi d'un devenir voué à la mort, ils trouvent dans l'immobilité de la tombe l'unité à laquelle ils aspiraient. Ce paradoxe (qui n'en est pas un) consiste à trouver "en bas", dans le trou ou dans le puits de la mort, ce que l'on cherchait en haut, au sommet de la tour ou de la flèche dressée.

Les héros qui se minéralisent illustrent en fait la trajectoire, fatale (c'est-à-dire déterminée) des corps en chute libre, statues ou stateurs, symboles d'une statique tandis que les héros qui choisissent l'écart font dévier cette trajectoire par un effort de volonté qui devient voyeurisme ou volupté. Cette déclinaison est le contraire du principe de l'inertie et symbolise le mouvement libre du vivant: "Paulum tantum quod momen mutatum dicere possis: les atomes, en chute libre dans le vide, s'écartent de leur trajectoire droite 'aussi peu qu'il soit possible de dire, par-là, que le mouvement s'en trouve modifié" (Serres 1977, 10). Cet écart, cette différentielle n'est autre que le clinamen ou le "secret dernier de la décision d'un sujet, son inclination" (Serres 1977, 10).

\section{BIBLIOGRAPHIE}

Barthes, Roland, Sur Racine, Paris : Éditions du Seuil, 1963.

Beatty, C. J. P., "Desperate Remedies", Thomas Hardy Yearbook 2 (1971) : 29-38.

Deleuze, Gilles et Guattari, Félix, Mille Plateaux, Paris : Éditions de Minuit, 1980.

Efron, Arthur, "The Tale, the Teller, and Sexuality in Tess of the Urbervilles", Punch 28 (1967) :

$55-80$.

Encyclopédie thématique. Sciences, vol. 3., "Magnétisme”, Paris : Encyclopædia Universalis, 2005.

Escuret, Annie, "Le macabre dans l'œuvre romanesque de Thomas Hardy”, Cahiers Victoriens et Édouardiens 15 (avril 1982) : 91-107.

Hardy, Thomas, The Complete Poems, ed. James Gibson, Basingstoke : Palgrave, 2001.

Hardy, Thomas, Desperate Remedies, London : Penguin Classics, 1998.

Hardy, Thomas, Far from the Madding Crowd, London : Penguin Classics, 2003.

Hardy, Thomas, Jude the Obscure, London : Penguin Classics, 1998.

Hardy, Thomas, The Life and Work of Thomas Hardy, ed. Michael Millgate, London : Macmillan, 1989. 
Hardy, Thomas, The Mayor of Casterbridge, London : Penguin Classics, 2003.

Hardy, Thomas, A Pair of Blue Eyes, London : Penguin Classics, 1998.

Hardy, Thomas, The Pursuit of the Well-Beloved and The Well-Beloved, London : Penguin Classics, 1997.

Hardy, Thomas, Le Retour au pays natal, trad. Marie Canavaggia, introd. Jean-Jacques Mayoux, Paris : Nouvelles Éditions Latines (“Les Maîtres Étrangers”), 1947.

Hardy, Thomas, The Return of the Native, London : Penguin Classics, 1999.

Hardy, Thomas, Tess of the D'Urbervilles, London : Penguin Classics, 2003.

Hardy, Thomas, Under the Greenwood Tree, London : Penguin Classics, 2004.

Hardy, Thomas, The Woodlanders, ed. Dale Kramer, Oxford : Clarendon Press, 1981.

Hardy, Thomas, The Woodlanders, London : Penguin Classics, 1998.

Holland, Norman, “Jude the Obscure : Hardy's Symbolic Indictment of Christianity”, NineteenthCentury Fiction 9 (June 1954) : 50-60.

Horne, Lewis B., "The Darkening Sun of Tess Durbeyfield", Texas Studies in Literature and Language 13.2 (Summer 1971) : 299-311.

Jallat, Jeannine, “Le masque ou l'art du déplacement”, Poétique 8 (1971) : 479-88.

Miller, Joseph Hillis, Thomas Hardy : Distance and Desire, Cambridge, Mass. : Harvard University Press, 1971.

Proust, Marcel, À la Recherche du temps perdu, III. La Prisonnière, La Fugitive, Le Temps Retrouvé, Paris : Gallimard ("Bibliothèque de la Pléiade"), 1954.

Purdy, Richard Little and Millgate, Michael eds., The Collected Letters of Thomas Hardy. Volume II : 1893-1901, Oxford : Clarendon Press, 1980.

Rosset, Clément, Logique du pire, Paris : PUF, 1971.

Rousset, Jean, Forme et signification, Paris : Librairie José Corti, 1962.

Serres, Michel, Feux et signaux de brume : Zola, Paris : Grasset, 1975.

Serres, Michel, Genèse, Paris : Grasset, 1982.

Serres, Michel, La Naissance de la physique dans le texte de Lucrèce. Fleuves et turbulences, Paris : Éditions de Minuit, 1977.

Starobinski, Jean, L'Eil vivant, Paris : Gallimard, 1961.

Zabel, Morton Dauwel, "Hardy in Defense of His Art : The Aesthetic of Incongruity", Craft and Character: Texts, Method, and Vocation in Modern Fiction, New York : The Viking Press, 1957. Article paru une première fois dans Southern Review 6 (1940) : 125-49.

\section{NOTES}

1. Il est arbitraire d'isoler au départ une partie de l'œuvre. Certes, il est difficile de tout étudier mais, lorsque nous avons choisi notre champ d'investigation, la poésie de Hardy constituait déjà le domaine de recherche de notre collègue Jean Vaché. Le chiffre "quinze" (pour les romans) peut paraître contestable car The Poor Man and the Lady ne fut jamais publié. Dans la mesure où "An Indiscretion in the Life of an Heiress" a été composé à partir du manuscrit de The Poor Man and the 
Lady, nous avons décidé de le placer parmi les romans (et non parmi les nouvelles). Pour nous, Hardy aura donc écrit quinze romans et non quatorze, qui est le chiffre habituellement cité.

2. Il est difficile de ne pas songer à d'autres invites similaires comme le titre du célèbre roman de Samuel Butler: The Way of all Flesh. La petite histoire nous apprend que le chien de Hardy se nommait "Wessex". Beau nom pour un animal domestique! Certes, cela peut nous faire sourire ; mais, dans la mesure où ce terme joue le même rôle que celui de "Yoknapatawpha" chez Faulkner, on peut se demander pourquoi le chien (l'“enfant" chéri du couple stérile) fut ainsi baptisé. Wessex est un dénominateur commun des romans, de la famille, de l'arbre, de la carte aux multiples espaces où l'utérus, le lit et le tombeau se confondent. La géographie du Wessex a fait couler beaucoup d'encre mais nous pensons que le chien fait partie de la "famille" parce qu'il est un membre parodique de l'humanimalité hardyenne - l'incarnation d'un devenir-animal raté - substitut d'un enfant qui ne vint jamais.

3. Notre thèse date de 1983 , et à cette époque, les éditions critiques des romans de Hardy n'étaient pas encore publiées, à l'exception précisément de celle que Dale Kramer a consacrée à The Woodlanders, et à laquelle nous nous référons ici (Oxford : The Clarendon Press, 1981).

4. L'absurdité d'une telle démarche est soulignée dans la préface de The Return of the Native de juillet 1895 et dans l'ajout daté d'avril 1912 (Hardy 1999, 429). Le même thème est repris avec humour dans la Préface de The Woodlanders, qui porte par ailleurs la même date que cet ajout (Hardy 1998d, 368-369). L'“anti-réalisme” de Hardy est du reste étudié par Morton Dauwel Zabel dans son article "Hardy in Defense of His Art : The Aesthetic of Incongruity".

5. En ce qui concerne le tragique, nous renvoyons le lecteur à l'ouvrage remarquable de Clément Rosset, Logique du pire, dans la mesure où nous pensons comme lui que la vision tragique est une vision du multiple qui, poussée à l'extrême, devient vision de rien, c'est-à-dire de rien que ce soit (dans l'ordre du pensable).

6. Hardy n'est en aucune façon le porte-parole d'un déterminisme. L'impressionnisme de sa technique est une mise en cause de tout système pré-établi ainsi qu'il le souligne dans la préface de Jude the Obscure d'août 1895 : "Like former productions of this pen, Jude the Obscure is simply an endeavour to give shape and coherence to a series of seemings, or personal impressions, the question of their consistency or their discordance, of their permanence or their transitoriness, being regarded as not of the first moment." (Hardy 1998b, 3-4) Dans la mesure où la prudence du "voyeur" est offerte comme alternative, il n'y a pas à proprement parler d'enchaînement fatal pré-établi mais simple erreur de jugement/vision dans le choix de départ car l'homme est libre de faire "le bien" en se tenant à distance ou de causer sa perte en se ruant sur une proie insaisissable.

7. Cette brève analyse du motif thématique de la "fem-être" pourrait être complétée par une étude de tout ce qui remplit une fonction analogue. La fenêtre qui frustre joue le même rôle que les chambres séparées, les murs, les lettres ou les photographies fétiches comme celles que Sue sème sur son passage. Notre propos, toutefois, n'est pas de dresser un inventaire mais de signaler un fonctionnement en prenant un exemple parmi d'autres.

8. Voir à ce sujet l'article de Lewis B. Horne, "The Darkening Sun of Tess Durbeyfield", qui offre une étude assez brève de la cosmisation des rapports dans Tess. On trouvera également quelques bonnes remarques sur ce même sujet dans l'article de Arthur Efron, "The Tale, the Teller, and Sexuality in Tess of the Urbervilles".

9. Pour plus de détails, nous renvoyons le lecteur à notre article "Le macabre dans l'œuvre romanesque de Thomas Hardy", où nous opposons les images du grouillement, de la décomposition et de la putréfaction aux images marmoréennes faisant intervenir la pierre sous toutes ses formes et qui renvoient à une paralysie définitive (par opposition au premier groupe d'images qui participent du cycle végétal et renvoient au devenir cyclique).

10. Dans un article intitulé "Le masque ou l'art du déplacement", Jeannine Jallat attire notre attention sur les textes critiques qui font appel à des "images d'espace" : "la dialectique du même 
et de l'autre, de l'identité et de la différence s'est, dans la critique contemporaine, singulièrement spatialisée et [...] de tous les horizons, nos méthodologies $\mathrm{du} \mathrm{XX}^{\mathrm{e}}$ siècle $\mathrm{y}$ font un recours constant. La figure de l'écartement, la voie oblique, la structure triangulaire du détour et toute une topologie de l'inclusion et de l'exclusion font un 'espace critique' où se retrouvent J. P. Richard et G. Genette, Starobinski, Levi-Strauss et R. Girard, M. Foucault et M. Serres. [...] Il y a là trop de rencontres pour qu'il soit sans intérêt d'en interroger un jour la problématique" (Jallat 487).

11. “[D]evenir n'est pas une évolution, du moins une évolution,par descendance et filiation. Le devenir ne produit rien par filiation, toute filiation serait imaginaire. Le devenir est toujours d'un autre ordre que celui de la filiation. Il est de l'alliance. Si l'évolution comporte de véritables devenirs, c'est dans le vaste domaine des symbioses qui met en jeu des êtres d'échelles et de règnes tout à fait différents, sans aucune filiation possible. [...] Si le néo-évolutionnisme a affirmé son originalité, c'est en partie par rapport à ces phénomènes où l'évolution ne va pas d'un moins différencié à un plus différencié, et cesse d'être une évolution filiative héréditaire pour devenir plutôt communicative ou contagieuse." (Deleuze et Guattari 291-292)

\section{INDEX}

Mots-clés : roman, regard, désir, eros, fenêtre, Wessex, microcosme, macrocosme

Keywords : novel, gaze, desire, Eros, window, Wessex, microcosm, macrocosm oeuvrecitee Desperate Remedies, Far from the Madding Crowd, Jude the Obscure, Laodicean (A), Mayor of Casterbridge (The), Moments of Vision, Pair of Blue Eyes (A), Pink Frock (The), Return of the Native (The), Roman Road (The), Tess of the d'Urbervilles, Time's Laughingstocks, Trumpet-Major (The), Two on a Tower, Under the Greenwood Tree, Well-Beloved (The), Woodlanders (The) 